\title{
On the Empirical Equivalence between Special Relativity and Lorentz's Ether Theory
}

\author{
Pablo Acuña L. \\ Institute for History and Foundations of Science, Utrecht University \\ Budapestlaan 6, 3584 CD, Utrecht \\ p.t.acunaluongo@uu.nl
}

\begin{abstract}
In this paper I argue that the case of Einstein's special relativity vs. Hendrik Lorentz's ether theory can be decided in terms of empirical evidence, in spite of the predictive equivalence between the theories. In the historical and philosophical literature this case has been typically addressed focusing on non-empirical features (non-empirical virtues in special relativity and/or non-empirical flaws in the ether theory). I claim that non-empirical features are not enough to provide a fully objective and uniquely determined choice in instances of empirical equivalence. However, I argue that if we consider arguments proposed by Richard Boyd and by Larry Laudan and Jarret Leplin, a choice based on non-consequential empirical evidence favoring Einstein's theory can be made.
\end{abstract}

Keywords: relativity; Einstein; Lorentz; empirical equivalence, empirical evidence, non-empirical virtues.

\section{Introduction}

Albert Einstein presented his special theory of relativity in 1905. As it is widely known, the adoption of this theory implied a revolutionary change in our views of space and time. Not so widely known is the fact that in 1904 the Dutch physicist Hendrik Lorentz proposed a theory that accounted for the same corpus of phenomena that Einstein's theory explained, but that was grounded on a conceptual framework in which the description of space and time was the traditional, Newtonian one. In 1905-6 Henri Poincaré introduced some amendments in Lorentz's theory that resulted in the full predictive equivalence between this theory and special relativity. Nowadays, Lorentz's ether theory is interesting only for historical and conceptualphilosophical reasons - the theory was definitively abandoned by the scientific community ca.1911.

The question of why was special relativity accepted and Lorentz's theory rejected is very interesting both from a historical and a conceptual point of view. An account of the particular reasons that physicists of the time invoked to make their choice is a necessary ingredient for any accurate and complete historical description of one of the most important episodes in the history of physics. On the other hand, since the case of special relativity vs. Lorentz's ether theory constitutes an example of the general problem of empirical equivalence and underdetermination of theory choice, this case is quite important from a philosophical standpoint as well. 
Thus, it is not surprising that the case of Einstein vs. Lorentz has received a good deal of attention in the historical and philosophical literature. What all of the arguments that intend to justify a choice favoring Einstein's theory have in common is that they are grounded on non-empirical features-non-empirical virtues in special relativity and/or non-empirical flaws in Lorentz's theory. In this paper I challenge this type of arguments. I claim that, by their very nature, non-empirical features cannot be invoked to justify a uniquely determined choice in cases of empirical equivalence. But this does not mean that the specific example of empirical equivalence at issue cannot be decided. Using two patterns of theory confirmation based on non-consequential empirical evidence introduced by Richard Boyd (1973) and Larry Laudan and Jarret Leplin (1991), I argue that the case of Lorentz vs. Einstein can be evidentially decided in a unique way. I also maintain that reference to one of these models (Boyd's) allows us to make sense of the case not only from a conceptual point of view, but also from a historical stance.

This paper is divided in four sections. In section 2 I offer an outline of the essentials of Lorentz's ether theory, paying special attention to the contributions and amendments introduced by Henri Poincaré which are crucial to obtain a full predictive equivalence with respect to special relativity, and to the features of the theory that can be considered as relevant to make a choice ${ }^{1}$. In section 3 I present and assess the most influential arguments that have been offered to decide the case. As I just mentioned, all of these arguments are of a non-empirical nature. In section 4 I explain why non-empirical features cannot count as a fully satisfactory solution for the problem of empirical equivalence in general, and for the case of Lorentz vs. Einstein in particular. In section 5 I explain and defend the patterns of non-consequential empirical evidence proposed by Boyd and by Laudan and Leplin, and then apply them to the case at issue. I conclude that such patterns provide two strong arguments that allow a uniquely determined choice favoring Einstein's theory, and that one of these arguments enables us to make sense of the main historical reason why special relativity prevailed.

\section{Lorentz's (and Poincaré's) theory ${ }^{2}$}

In 1892 Lorentz published two works that contain the main postulates of his ether theory. In The Electromagnetic Theory of Maxwell and its Application to Moving Bodies, he introduced its first conceptual cornerstone: a set of coordinate transformations. Lorentz showed that Maxwell's equations-which at the time were thought to hold in the ether-rest frame-retain their form in frames in motion through the ether if, instead of the Galilean transformations, the coordinate transformations he introduced were applied. The specific form of these transformations is the following:

\footnotetext{
${ }^{1}$ Special relativity is a widely known theory, so, for reasons of space, I will not offer any explanation of it. For the reader which is not familiar with this theory, good and technically accessible sources are (Sartori 1996) and (Rindler 1991). For a deeper treatment of Einstein's theory, see, for example, (Miller 1998) and (Torretti 1996).

${ }^{2}$ In the following presentation of Lorentz's theory I closely follow Michel Janssen's (1995) excellent historical and conceptual work. (Miller 1998) is also a good source.
} 


$$
\mathbf{x}^{\prime}=\mathbf{x}=\mathbf{x}_{0}-\mathbf{v} t_{0} \quad t^{\prime}=t-\left(v / c^{2}\right) x=t_{0}-\left(v / c^{2}\right) x
$$

where the primed quantities belong to the auxiliary frame $S^{\prime}$ which moves with velocity $v$ with respect to frame $S_{0}$ at rest in the ether, the unprimed quantities belong to a real, Galilean frame $S$ which moves with velocity $v$ with respect to $S_{0}$, and the quantities with subscript 0 belong to the frame $S_{0}$.

The crucial quantity in the transformations was $t^{\prime}$, better known as 'local time'. With this mathematical tool—he did not assign any physical meaning to it at this stage — Lorentz showed that, up to first order of $v / c$, Maxwell's equations are invariant. Thus, the predicted result of the theory for most of the optical etherdrift experiments that had been proposed and performed was negative- predictions which were consistent with the observed results. Based on these coordinate transformations, Lorentz formulated his famous theorem of corresponding states:

If there is a solution of the source free Maxwell equations in which the real field $\mathbf{E}$ and $\mathbf{B}$ are certain functions of $\mathbf{x}_{0}$ and $t_{0}$, the coordinates of $S_{0}$ and the real Newtonian time, then if we ignore terms of order $v^{2} / c^{2}$ and smaller, there is another solution of the source free Maxwell equations in which the fictitious field $\mathbf{E}^{\prime}$ and $\mathbf{B}^{\prime}$ are those same functions of $\mathbf{x}^{\prime}$ and $t^{\prime}$, the coordinates of $S^{\prime}$ and local time. (Janssen 1995, section 3.1.1).

In spite of the predictive success of Lorentz's theory in explaining the result of many ether-drift tests, it was not able to explain the negative result of the Michelson-Morley experiment - which was sensitive enough in order to detect effects of order $v^{2} / c^{2}$. In a nutshell, this experiment was designed to measure the effect of the motion of the earth through the ether on the velocity of light. Such effect implies that the time it takes a light beam to travel along the arm of the interferometer which lies along the direction of motion through the ether, and the time it takes for a beam of light to travel along the arm which is perpendicular to the direction of motion, are different. This difference yields an interference pattern that, as the interferometer gets rotated, should change. However, no such changes were observed, everything happened as if the time of the two trips were the same. This result could be readily explained by assuming that the Earth carries along an ether-atmosphere in its translational motion, but Lorentz himself had shown in 1886 that G. G. Stokes' theory — which assumed this kind of ether-drag — was inconsistent.

In a second work he published in 1892, entitled The Relative Motion of the Earth and the Ether, Lorentz introduced a further hypothesis in order to account for the negative result of the Michelson-Morley experiment. He simply argued that if a length-contraction in the direction of motion through the ether affected physical bodies, then the predicted time for the trip of the light beams across the arms of the interferometer would be the same - and under this assumption the Michelson-Morley experiment yields a null result. Though the length-contraction hypothesis was clearly introduced with the specific goal of explaining one particular experiment, Lorentz did provide a plausibility argument to support it. By the time it was known that electromagnetic forces $\mathbf{F}$ in a frame at rest in the ether and the electromagnetic forces $\mathbf{F}^{\prime}$ in a system in motion with respect to the ether are related in the following way: 


$$
F^{\prime}{ }_{x \prime}=F_{x} \quad F_{y^{\prime}}^{\prime}=\frac{F_{y}}{\sqrt{1-v^{2} / c^{2}}}=\gamma F_{y} \quad F_{z^{\prime}}^{\prime}=\frac{F_{z}}{\sqrt{1-v^{2} / c^{2}}}=\gamma F_{z} .
$$

This result can be interpreted as stating that if a system at rest is in equilibrium under a configuration of forces $\mathbf{F}$, when in motion through the ether that same system is in equilibrium under a configuration of forces $\mathbf{F}^{\prime}$. Lorentz then argued that if the 'molecular forces' that determine the shape and length of bodies also transform in this way, then objects in motion through the ether get contracted by a factor $\sqrt{1-v^{2} / c^{2}}-$ which is exactly the factor required for the prediction of a negative result in the Michelson-Morley experiment. It is important to mention that in 1889 , G. F. Fitzgerald had already proposed this same lengthcontraction hypothesis in order to explain the result of the Michelson-Morley experiment, and the plausibility argument he introduced for the hypothesis was equivalent to the one that Lorentz proposed in 1892. For this reason the contraction is also known as the Lorentz-Fitzgerald contraction ${ }^{3}$.

Lorentz presented all these results - the coordinate transformations and the length-contraction hypothesis - in a more systematic and structured way in his 1895 Attempt of a Theory of Electric and Optic Phenomena in Moving Bodies. This work rapidly became a central point of discussion for the scientific community of the time. Henri Poincare introduced observations and criticisms that were important for its further development. He complained that the explanations for the negative results of ether-drift experiments of first and second order the theory provided were two different and logically disconnected parts of the theory. In 1900 he stated that 'the same explanation must be found for the two cases, and everything tends to show that this explanation would serve equally well for the terms of higher order and that the mutual destruction of these terms will be rigorous and absolute' (Poincaré 1900, p. 172).

A second important contribution of Poincaré on Lorentz's theory was to elucidate that 'local time' was not only a mathematical tool, as Lorentz originally conceived, but that it contained a physical meaning. In a contribution for a volume celebrating the $25^{\text {th }}$ anniversary of Lorentz's doctorate, Poincare explained his point by means of an illustration that strikingly resembles Einstein's method for the synchronization of clocks. He showed that if two observers at rest with respect to each other, but in motion with respect to the ether, try to synchronize their clocks by means of light pulses, the result is that their synchronized clocks are late with respect to the real time. Poincaré went one step further than Lorentz, 'local time' was not merely a mathematical tool that allowed the introduction of an auxiliary frame in which Maxwell's equations are invariant. The explanation of the negative results of (first order of $v / c$ ) optic experiments was thus associated to 'local time' as the time measured in a frame moving with respect to the ether:

In fact, they mark the same hour at the same physical instant, but on the one condition, that the two stations are fixed [with respect to the ether]. Otherwise the duration of the transmission will not be the same in the two senses, since the station $A$, for example, moves forward to meet the optical perturbation emanating from $B$, whereas the station $B$ flees before the perturbation emanating from $A$. The watches adjusted in that way

\footnotetext{
${ }^{3}$ See (Hunt 1988) and (Brown 2001, 2003).

${ }^{4}$ For an excellent treatment of Poincaré's involvement in the development of Lorentz's ether theory, see (Darrigol 1995). (Miller 1998) is also useful.
} 
will not mark, therefore, the true time; they will mark what may be called the local time, so that one of them will gain on the other. It matters little, since we have no means to perceive it. All the phenomena which happen at $A$, for example, will be late, but all will be equally so, and the observer will not perceive it, since his watch is slow; so, as the principle of relativity would have it, he will have no means of knowing whether he is at rest or in absolute motion. (Poincaré 1904, p. 99).

Finally, in the same contribution for Lorentz's doctorate anniversary, Poincaré showed that Lorentz's theory implied a violation of Newton's third law. The ether postulated by the theory electrodynamically affected the behavior of physical objects - the length-contraction was grounded on the interaction of bodies with the ether, for example. However, according to the theory material bodies did not affect the ether in any way. Moreover, Poincaré also noticed that this violation was connected to a violation of the law of momentum conservation and of the center of mass theorem. Poincaré illustrated his point by means of an example:

Imagine, for example, a Hertzian oscillator, like those used in wireless telegraphy; it sends out energy in every direction; but we can provide it with a parabolic mirror, as Hertz did with his smallest oscillators, so as to send all the energy produced in a single direction. What happens then according to the theory? The apparatus recoils as if it were a cannon and the projected energy a ball; and that is contrary to the principle of Newton since our projectile has no mass, it is not matter, it is energy. (Poincaré 1904, p. 101).

If the recoil of the Hertzian antenna is not accompanied by a reaction in the ether, Newton's third law is violated. If this reaction does not happen, there is a loss of momentum in the system that is not compensated. Finally, the recoil of the antenna also implies that the center of mass of the system gets accelerated. Poincaré solved the three problems by one single theoretical stroke: the introduction of a 'fictitious fluid' in the ether. He assigned this fluid an electromagnetic momentum given by $\frac{1}{4 \pi c} \int E \times B d V$, where $V$ stands for the volume occupied by the fluid. This momentum played the role of the reaction for the recoil of the antenna, so that $\frac{d}{d t}\left[m \mathbf{v}+\frac{1}{4 \pi c} \int E \times B d V\right]=0$. Both Newton's third law and momentum conservation are satisfied if the fictitious fluid is considered.

The center of mass theorem could also be saved. The momentum expression for the fictitious fluid implicitly states that it possesses a mass density given by $E / c^{2}$, where $E$ stands for the electromagnetic energy carried by the fluid. If no electromagnetic energy is created or destroyed, the mass density of the fictitious fluid is conserved and the global center of mass of matter and fluid moves along a straight line and with constant velocity. If electromagnetic energy was destroyed and transformed into another form of energy, Poincaré had to assume that the fluid associated to the destroyed energy was not really destroyed, but brought to rest and 'stored' at the place of its destruction. In the case of electromagnetic energy created, the required assumption was that 'latent' fluid available at the place of that creation got liberated.

Two things are noteworthy regarding Poincaré's fictitious fluid. First, it implicitly included the energymass relation $E=m c^{2}$, but Poincaré did not have the theoretical framework required to recognize it in its 
full meaning ${ }^{5}$. Second, the physical underpinning of the fictitious fluid was rather strange. Poincaré introduced this quantity with the intention of clarifying how Lorentz's theory violated the Newtonian principles, and as a sort of desperate maneuver involving physical fictions in order to save them. Actually, Lorentz rejected the fictitious fluid insofar as it implied a kind of motion in the ether, which he believed to be totally immobile. However, this unease got softened when Max Abraham, in 1902, (re)introduced the concept of electromagnetic momentum as carried not by a fluid in the ether, but by the electromagnetic fields themselves. Electromagnetic momentum would later be a crucial feature of Lorentz's model of the electron in the definitive formulation of the ether theory.

The next step in the development of the theory was taken by Lorentz in his 1899 Simplified Theory of Electrical and Optical Phenomena in Moving Bodies. In this work, the definitive versions of the coordinate transformations and of the theorem of corresponding states were introduced. These definitive formulations implied that the Lorentz-invariance of Maxwell's equations was complete- the first order of $v / c$ restriction was not required any more. The specific form of the definitive transformations was the following:

$$
x^{\prime}=\gamma l\left(x_{0}-v t_{0}\right) \quad y^{\prime}=l y \quad z^{\prime}=l z \quad t^{\prime}=\gamma l\left[t_{0}-\left(v / c^{2}\right) x_{0}\right]^{6}
$$

As Michel Janssen has shown (1995, section 3.3.3), the fact that these transformations include the factor $\gamma={\sqrt{1-v^{2} / c^{2}}}^{-1}$ does not imply that they already contain the length-contraction effect, this effect requires a further physical assumption. Without it, the transformations are merely mathematical tools that define an auxiliary frame in which Maxwell's equations retain their ether-rest form. The assumption needed to assign a physical meaning to the transformations is what Janssen dubs the generalized contraction hypothesis:

If a material system, i.e., a configuration of particles, with a charge distribution that generates a particular electromagnetic field configuration in $S_{0}$, a frame at rest in the ether, is given the velocity $\mathbf{v}$ of a Galilean frame $S$ in uniform motion through the ether, it will rearrange itself so as to produce the configuration of particles with a charge distribution that generates the electromagnetic field configuration in $S$ that is the corresponding state of the original electromagnetic configuration in $S_{0}$. (Janssen 1995, section 3.3.3, my emphasis).

In other words, under the generalized contraction hypothesis, the factor $\gamma$ does express a physical effect, and therefore, under this assumption the transformations acquire a physical meaning. Without it, the meaning

\footnotetext{
${ }^{5}$ Actually, in a 1906 derivation of $E=m c^{2}$ —based on the observance of the center of mass theorem-Einstein explicitly referred to Poincaré's 'fictitious fluid' argument. For an explanation of Einstein's 1906 derivation and a comparison with Poincaré's argument, see (Janssen 2003).

${ }^{6}$ Lorentz applied the same three-step method he used in 1892. That is, the transformations involved three reference frames. Frame $S_{0}$ is at rest in the ether, $S$ is a Galilean frame moving with velocity $v$ with respect to $S_{0}$, and $S^{\prime}$ is an auxiliary frame that also moves with velocity $v$ with respect to $S_{0} . S_{0}$ and $S$ are connected by the Galilean transformations, whereas $S$ and $S^{\prime}$ are connected by the transformations $x^{\prime}=\gamma l x, y^{\prime}=l y, z^{\prime}=l z, t^{\prime}=l\left[t / \gamma-\gamma\left(v / c^{2}\right) x\right]$. Combining these two transformations we obtain the transformations connecting $S_{0}$ and $S^{\prime}$.

The term $l$ stands for a possible transverse deformation consistent with the negative result of the Michelson-Morley experiment. Lorentz set it to 1 (no transverse deformation) in 1904 for dynamical reasons we will see below. For a treatment of the history and meaning of $l$, see (Brown 2001) and (Janssen and Mecklenburg 2007).

The definitive version of the theorem of corresponding states is simply the same as in 1895 , but deleting the clause expressing the restriction to first order of $v / c$.
} 
of the transformation would be analogous to the mathematical tool interpretation that Lorentz offered for 'local time' in 1892-5.

The new version of the coordinate transformations, interpreted under the generalized contraction hypothesis, entails a very surprising result from the point of view of classical physics, namely, the velocitydependence of mass. I will provide only a brief sketch of Lorentz's reasoning. He considered an electron oscillating in an ether-rest frame, so the oscillation obeys Newton's second law $\mathbf{F}=m \mathbf{a}$. Then he considered the same electron, but now in a frame in motion with respect to the ether. In terms of the auxiliary quantities $\mathbf{x}^{\prime}$ and $t^{\prime}$, the state of motion of the electron in the moving frame is the same as its corresponding state of motion in the ether-rest frame. This implies that in terms of the real, ether-rest quantities the electron satisfies Newton's second law only if its mass depends on its velocity.

Remember that Lorentz assumed that all forces transform as electromagnetic ones do-in the definitive formulation of the theory this assumption is grounded on the generalized contraction hypothesis and the exact theorem of corresponding states-, so in a frame $S^{\prime}$ in motion with respect to the ether, the force $\mathbf{F}^{\prime}$ is given by

$$
F^{\prime}{ }_{x^{\prime}}=F_{x} \quad F^{\prime}{ }_{y^{\prime}}=\gamma F_{y} \quad F_{z^{\prime}}^{\prime}=\gamma F_{z}
$$

The relation between the acceleration of the electron in the ether-rest frame and in the moving frame-if the velocity and amplitude of the electron's oscillation are small enough as to be neglected - is given by

$$
a_{x^{\prime}}^{\prime}=\gamma^{3} a_{x} \quad a_{y^{\prime}}^{\prime}=\gamma^{2} a_{y} \quad a_{z^{\prime}}^{\prime}=\gamma^{2} a_{z}
$$

Considering the expression for the transformation of force above, Newton's second law can be formulated, in terms of the real, ether-rest quantities, in the following way:

$$
F_{x}=m \gamma^{3} a_{x} \quad F_{y}=m \gamma a_{y} \quad F_{z}=m \gamma a_{z}
$$

From these formulas it follows that if Newton's law is to hold in the moving frame, then the mass of the electron has to depend on its velocity-on the parameter $v$ in the factor $\gamma$. Assuming that the motion of the electron is only along the $x$-direction, the expressions for the longitudinal and transverse inertial mass, respectively, are

$$
m_{L}=m_{0} \gamma^{3} \quad m_{T}=m_{0} \gamma
$$

Lorentz provided an explanation of this surprising result by means of the model of the electron he introduced in his 1904 Electromagnetic Phenomena in Systems Moving with any velocity Less than that of Light. This model, in turn, was closely connected to an ambitious program that is generally known as the electromagnetic view of nature. The main goal of this program was to reduce all physics to 
electromagnetism. Its manifesto was introduced in 1900 by Wilhelm Wien, and it stated that the main tenet of this revolutionary framework for the development of physics was the assumption of an ontology determined by charged particles constituting all ponderable matter, whose inertial mass is of an entirely electromagnetic origin. That is, according to the electromagnetic view of nature, the inertial mass of any physical object is the outcome of the interaction between charges, their associated electromagnetic field, and the ether ${ }^{7}$.

Lorentz's 1904 model of the electron was given by five main tenets: i) a spherical electron that when in motion across the ether undergoes a physical deformation and becomes an ellipsoid - the deformation is described by the generalized contraction hypothesis and the coordinate transformations-;ii) all forces transform in the same way as electromagnetic ones do; iii) the origin of the entire inertial mass is of electromagnetic origin; iv) the deformation occurs only in the dimension parallel to the direction of motion; and v) the inertial mass of all bodies, charged or not, depends on velocity in the same way as the inertial mass of the electron does. It is clear that these assumptions heavily rely on the essential postulates of the electromagnetic worldview.

More specifically $^{8}$, if we take Newton's second law $\mathbf{F}=m \mathbf{a}$ under the assumption that all inertial mass is of electromagnetic origin - and therefore all mechanical mass supervenes on electromagnetic interactions - the law reduces to $\mathbf{F}=0$. Expressed in terms of momentum variation through time, the law becomes $\frac{d \mathbf{P}_{t o t}}{d t}=0$ (the subscript simply indicates that momentum need not be only mechanical), an expression for momentum conservation. By defining $\mathbf{F}_{\text {ext }}$ as the Lorentz-force acting on the electron coming from the external field, and $\mathbf{F}_{\text {self }}$ as the Lorentz-force coming from the electron self-field, the equation of motion for an electron in an external field that Lorenz stated was $\mathbf{F}_{\text {ext }}+\mathbf{F}_{\text {self }}=0$. The Lorentz-force on the electron from its self-field can be written as minus the derivative of electromagnetic momentum with respect to time, i.e., $\mathbf{F}_{\text {self }}=-\frac{d \mathbf{P}_{e m}}{d t} 9$, and given the equation of motion for the electron in an external field, it follows that $\mathbf{F}_{\text {ext }}=\frac{d \mathbf{P}_{e m}}{d t}$.

Assuming that the momentum is in the direction of motion, its formula can be written as $\mathbf{P}_{e m}=$ $\left(\frac{P_{e m}}{v}\right) \mathbf{v}=P_{e m} \frac{\mathbf{v}}{v}$. Differentiating this expression with respect to time we get $\frac{d \mathbf{P}_{e m}}{d t}=\frac{d P_{e m}}{d t} \frac{\mathbf{v}}{v}+P_{e m} \frac{d}{d t}\left(\frac{\mathbf{v}}{v}\right)$. Since we can consider $P_{e m}$ as a function of $v$, the first term in the right hand side of the last equation can be expressed as $\frac{d P_{e m}}{d v} \frac{d v}{d t} \frac{\mathbf{v}}{v}=\frac{d P_{e m}}{d v} \mathbf{a}_{L}$, where $\mathbf{a}_{L}$ stands for the longitudinal acceleration with respect to the

${ }^{7}$ For an exposition of the history and conceptual contents of the electromagnetic view of nature, see (McCormmach 1970) and (Kragh 1999).

${ }^{8}$ In the following sketch of Lorentz's model of the electron, I closely follow (Janssen and Mecklenburg 2007).

9 'The Lorentz force that an electron moving through the ether at velocity $\mathbf{v}$ experiences from its self-field can be written as minus the time derivative of the quantity that Abraham proposed to call the electromagnetic momentum:

$$
F_{\text {self }}=\int \rho(\mathbf{E}+\mathbf{v} \times \mathbf{B}) d^{3} x=-\frac{d \mathbf{P}_{e m}}{d t} .
$$

In this expression $\rho$ is the density of the electron's charge distribution, and $\mathbf{E}$ and $\mathbf{B}$ are the electric and magnetic field produced by this charge distribution. The electromagnetic momentum of these fields is defined as

$$
P_{e m} \equiv \int \varepsilon_{0}(\mathbf{E} \times \mathbf{B}) d^{3} x
$$

and doubles as the electromagnetic momentum of the electron itself'. (Janssen and Mecklenburg 2007, p. 8). 
direction of motion. The second term can be written as $P_{e m} \frac{d}{d t}\left(\frac{\mathbf{v}}{v}\right)=\frac{P_{e m}}{v} \mathbf{a}_{T}$, where $\mathbf{a}_{T}$ stands for the transverse acceleration with respect to the direction of motion. Finally, since $\mathbf{F}=\frac{d \mathbf{p}}{d t}=m \mathbf{a}$, we have that $\frac{d \mathbf{P}_{e m}}{d t}=m_{L} \mathbf{a}_{L}+m_{T} \mathbf{a}_{T}$, with the longitudinal mass of the electron $m_{L}=\frac{d P_{e m}}{d v}$, and the transverse mass of the electron $m_{T}=\frac{P_{e m}}{v}$.

It can be shown that $\mathbf{P}_{e m}=\frac{4}{3} \gamma l\left(\frac{U_{e m}^{\prime}}{c^{2}}\right) \mathbf{v}^{10}$-where $U_{e m}^{\prime}$ stands for the energy of the electron in the frame $S^{\prime}$ in motion with respect to the ether, $c$ is the velocity of light, and $l$ stands for the transverse deformation factor mentioned above. Plugging the right hand side of this equation in the expressions for the electron masses, in the case of longitudinal mass we get $m_{L}=\frac{d(\gamma l v)}{d v} \frac{4}{3} \frac{U^{\prime} e m}{c^{2}}$, and since $\frac{d}{d v}(\gamma v)=\gamma^{311}$, then $m_{L}=y^{3} l \frac{4}{3} \frac{U^{\prime} \mathrm{em}}{c^{2}}$. In the case of the transverse mass, we get $m_{T}=\gamma l \frac{4}{3} \frac{U^{\prime} \mathrm{em}}{c^{2}}$. Since the expressions that Lorentz derived in 1899, from the theorem of corresponding states and the generalized contraction hypothesis applied to $\mathbf{F}=m \mathbf{a}$, are $m_{L}=y^{3} l m_{0}$ and $m_{T}=\gamma l m_{0}$, it turns out that for the 1904 results to be consistent with the 1899 expressions, the rest-mass must be defined as $m_{0}=\frac{4}{3} \frac{U^{\prime} \mathrm{em}}{\mathrm{c}^{2}}$, and the factor $l$ must be set to $1^{12}$.

Supporters of the electromagnetic worldview took the equation $\mathbf{F}_{\text {ext }}=\frac{d \mathbf{P}_{\text {em }}}{d t}=m_{L} \mathbf{a}_{L}+m_{T} \mathbf{a}_{T}$ as the fundamental equation of motion, and interpreted Newton's second law as special cases of it. For $v=0$, that is, for an electron at rest in the ether, it holds that $m_{L}=m_{T}=m_{0}$, so that for the ether-rest electron $\mathbf{F}_{\text {ext }}=$ $m_{0}$ a. For a moving electron, the values of $m_{L}$ and $m_{T}$ differ from $m_{0}$ only by an amount of the order $v^{2} / c^{2}$. Therefore, for $v \ll c, \mathbf{F}_{\text {ext }} \approx m_{0} \mathbf{a}$. This reduction of a central law of mechanics to electrodynamics was, of course, considered as a promising result of the electromagnetic program. Furthermore, as we just saw, from his model of the electron Lorentz obtained an expression of the velocity-dependence of inertial mass that was consistent with the expression he found in 1899. That is, he naturally interpreted that he had found a model of the elementary charged-particle that explained the results he obtained in 1899 without specific dynamical assumptions. In other words, the 1904 model of the electron provided strong support for the universal scope of the generalized contraction hypothesis. If all laws are, bottom-line, electromagnetic, then the Lorentzinvariance of all laws of physics is a natural consequence.

However, a big difficulty quickly came up. It turned out that the expression of the longitudinal mass of the electron in terms of its momentum was incompatible with the expression in terms of its energy. Consider an electron moving in the $x$-direction and assume the absence of an external field. The force the electron

${ }^{10}$ See (Janssen and Mecklenburg 2007, p. 19).

${ }^{11} \frac{d}{d v}(\gamma v)=\gamma+v \frac{d \gamma}{d v}=\gamma+y^{3} \beta^{2}$, with $\beta=\frac{v}{c}$. Since $\gamma=\gamma^{3}\left(1-\beta^{2}\right)$, then $\frac{d}{d v}(\gamma v)=\gamma^{3}\left(1-\beta^{2}+\beta^{2}\right)=\gamma^{3}$. From this result is easy to infer that $\frac{d \gamma}{d v}=\gamma^{3} \frac{v}{c^{2}}$, this expression will be useful below.

${ }^{12}$ Since $\frac{d}{d v}(\gamma v)=\gamma^{3}$ and $\frac{d \gamma}{d v}=\gamma^{3} \frac{v}{c^{2}}$, it follows that $m_{L}=\frac{d(\gamma l v)}{d v} \frac{4}{3} \frac{U^{\prime} e m}{c^{2}}=\left(y^{3} l+\gamma v \frac{d l}{d v}\right) \frac{4}{3} \frac{U^{\prime} e m}{c^{2}}$. For this expression to be equivalent to $m_{L}=y^{3} l m_{0}$, it must be the case that $\frac{d l}{d v}=0$, and since $l$ is a function of $v$ and can differ from 1 only by an amount of the order $v^{2} / c^{2}$, then $l=1$. This is the dynamical reason why Lorentz established that the deformation of the moving-across-the-ether electron is only longitudinal. With $l=1$, the coordinate transformations in Lorentz's theory are identical to the ones that Einstein obtained in 1905. 
experiences from its self-field does an amount of work that can be expressed in terms of the energy of the electron, $d U_{e m}=-d W=-\mathbf{F}_{\text {self }} \cdot d \mathbf{x}$. Substituting $-\mathbf{F}_{\text {self }}$ for $\frac{d \mathbf{P}_{e m}}{d t}$ we get $d U_{e m}=\frac{d \mathbf{P}_{e m}}{d t} \cdot d \mathbf{x}=m_{L} a_{L}$. $d \mathbf{x}=m_{L} \frac{d v}{d t} d x=m_{L} v d v$. From this last equation it follows that $m_{L}=\frac{1}{v} \frac{d U_{e m}}{d v}$.

The relation between the energy of an electron in an ether-rest frame and its energy in a moving frame is $U_{e m}=l\left(\frac{4 \gamma}{3}-\frac{1}{3 \gamma}\right) U_{e m}^{\prime}{ }^{13}$. If we set $l$ to 1 and plug the right hand side of this equation in $m_{L}=\frac{1}{v} \frac{d U_{e m}}{d v}$ we get $m_{L}=\frac{1}{v} \frac{d}{d v}\left(\frac{4 \gamma}{3}-\frac{1}{3 \gamma}\right) U_{e m}^{\prime}=\frac{1}{v} \frac{4}{3} \frac{d \gamma}{d v} U_{e m}^{\prime}-\frac{1}{3 v} \frac{d}{d v}\left(\frac{1}{\gamma}\right) U_{e m}^{\prime}$, and since $\frac{d \gamma}{d v}=\gamma^{3} \frac{v}{c^{2}}$, we finally obtain $m_{L}=\gamma^{3} \frac{4}{3} \frac{U_{e m}^{\prime}}{c^{2}}-\frac{1}{3 v} \frac{d}{d v}\left(\frac{1}{\gamma}\right) U_{e m}^{\prime}$. Notice that the first term of the right hand expression in this equation is equal to the value of the longitudinal mass of the electron obtained from its momentum. The presence of the second term is thus the root of the problem: longitudinal mass in terms of momentum is not equal to longitudinal mass in terms of energy. In Lorentz's model of the electron, we have that $m_{L}=\frac{d P_{e m}}{d v}$, that $m_{L}=\frac{1}{v} \frac{d U_{e m}}{d v}$, and that $\frac{d P_{e m}}{d v} \neq \frac{1}{v} \frac{d U_{e m}}{d v}$.

Max Abraham was the first to notice the inconsistency, and he stated that the problem came up because in Lorentz's theory the total energy of the electron could not be accounted for only in terms of the electromagnetic energy. Following this line of thought, Poincaré provided a solution in his 1906 On the Dynamics of the Electron ${ }^{14}$. He introduced a quantity that is commonly known as Poincaré-pressure, defined as $P_{p}=-\frac{1}{3} \frac{U^{\prime} \mathrm{em}}{V_{0}}$. This quantity contributes the missing energy $\frac{1}{3} \frac{U^{\prime} \mathrm{em}}{\gamma}$, which is minus the product of the $P_{p}$ and the volume of the moving electron $V_{0} / \gamma$. By adding this missing energy to $U_{e m}=l\left(\frac{4 \gamma}{3}-\frac{1}{3 \gamma}\right) U_{e m}^{\prime}$, we have that the total energy is $U_{t o t}=\frac{4}{3} \gamma U_{e m}^{\prime}$. Defining the longitudinal mass in terms of energy as $\frac{1}{v} \frac{d U_{t o t}}{d v}$, then $m_{L}=\frac{1}{v} \frac{d U_{t o t}}{d v}=\frac{1}{v} \frac{d \gamma}{d v} \frac{4}{3} U_{e m}^{\prime}=\gamma^{3} \frac{4}{3} \frac{U^{\prime} e m}{c^{2}}=\gamma^{3} m_{0}=\frac{d P_{e m}}{d v}$, so the inconsistency is solved.

The compensating pressure Poincaré introduced made Lorentz's electron stable, for it counterbalanced the repulsive Coulomb forces, that, without assuming $P_{p}$, would make the moving electron to explode. Besides, $P_{p}$ provided a physical foundation for the Lorentz contraction affecting the electron itself. The pressure is exerted only on the surface of the electron, so the forces involved make the electron to contract as it moves through the ether. Yet another nicety of $P_{p}$ is that, from a modern perspective, it solves the " $4 / 3$ puzzle' involved in the energy-mass relation implicit in Lorentz's theory. This feature is crucial for the case of Lorentz vs. Einstein. If the total energy of the electron were given by the electromagnetic energy, $m_{0}=$ $\frac{4}{3} \frac{U^{\prime} e m}{c^{2}}$ would be the definitive formula for inertial mass, so Lorentz's theory would not be empirically equivalent to special relativity and it would be falsified by the outcome of non-optical ether-drift experiments where the relativistic formula $E=m c^{2}$ is involved-the Trouton experiment, for example ${ }^{15}$. However, if we

\footnotetext{
${ }^{13}$ See (Janssen and Mecklenburg 2007, p. 18).

${ }_{15}^{14}$ For a detailed analysis of this work, see (Miller 1973).

${ }^{15}$ See (Janssen 2003).
} 
consider the missing energy contributed by $P_{p}$, the energy of the electron is $U_{\text {tot }}=\frac{4}{3} \gamma U^{\prime}{ }_{\text {em }}$, as we saw above, and therefore, $m_{0}=\frac{U_{t o t} 16}{c^{2}}$.

Notwithstanding the improvements of Lorentz's theory that $P_{p}$ allowed, there was a price that supporters of the electromagnetic program had to pay for its adoption. The quantity introduced by Poincaré was not electromagnetic. It contributed the missing non-electromagnetic energy required to keep the electron stable and to solve the inconsistency problem. Poincare and Lorentz simply opted for sacrificing the purity of the electromagnetic program. As we will see below, the impossibility of obtaining a full reduction of all the laws of physics to electrodynamics determines a non-empirical criterion feature that makes special relativity a better theory than Lorentz's.

In his 1906 work, Poincaré also corrected the charge and velocity transformations that Lorentz obtained. This correction is also crucial for the exact predictive equivalence between the ether theory and special relativity. In the case of the $x$-velocity transformation, Lorentz's formula was $u_{x}^{\prime}=\gamma u_{x}$, whereas the one that Poincaré obtained-which is identical to Einstein's - was $u_{x}^{\prime}=\frac{u_{x}-v}{1-v u_{x}}$, where $u$ is the velocity of an object in frame $S, u^{\prime}$ the velocity of the object in frame $S^{\prime}$, and $v$ the velocity of $S^{\prime}$ with respect to $S$. The problem, the French scientist noticed, was rooted in Lorentz's three-step method connecting the frames $S_{0}, S$ and $S^{\prime}$. Instead of applying a Galilean transformation to connect the real frame $S_{0}$ to the real frame $S$, and then a different transformation to connect the latter frame to the auxiliary frame $S^{\prime}$, Poincaré combined the successive transformations into a single one - which he called the Lorentz transformation - to directly connect the ether-rest frame to $S^{\prime}$. He considered the latter frame not as an auxiliary one, but as expressing measured quantities. However, just as Lorentz, Poincaré conceived the ether-rest quantities as the true ones ${ }^{17}$.

Another contribution that Poincare made to Lorentz's theory was a mathematical result: he showed that the Lorentz transformations form a group. A collection of transformations constitute a group if they are associative, if there exists an identity transformation, if the transformation obtained through two successive applications of a transformation in the collection is also a transformation in the collection, and if there exists an inverse transformation. By demonstrating the last property Poincaré showed that the Lorentz transformations are symmetrical ${ }^{18}$. Consider the frames $S$ and $S^{\prime}$ to be rotated in $180^{\circ}$ around their $y$-axis, so

\footnotetext{
${ }^{16}$ Herbert Ives (1952) has shown how the energy-mass relation could have been obtained, in its full meaning, from Poincaré's work on Lorentz's theory. That is, all the mathematic and physical machinery needed for the derivation was contained in Lorentz's theory as amended by Poincaré. Therefore, the predictive equivalence with respect to special relativity does not require that $E=m c^{2}$ has to be borrowed from Einstein's theory. Even though neither Lorentz nor Poincaré realized it, the equation was already there.

${ }^{17}$ See footnote 1 above. The transformations from $S$ to $S^{\prime}$, in their $d x$ and $d s$ form and with $l=1$ and choosing suitable units such that $c=1$, are $d x^{\prime}=\gamma d x$ and $d t^{\prime}=\frac{1}{\gamma} d t\left(1-\gamma^{2} v u_{x}\right)$. Thus, $u_{x}^{\prime}=\frac{d x^{\prime}}{d t \prime}=\frac{\gamma d x}{\frac{1}{\gamma} d t\left(1-y^{2} v u_{x}\right)}$. Since $\frac{d x}{d t}=u_{x}$, then $u_{x}^{\prime}=$ $\frac{\gamma^{2} u_{x}}{\left(1-\gamma^{2} v u_{x}\right)}$, and assuming $v \ll 1$, then $u_{x}^{\prime}=\gamma^{2} u_{x}$-this is a sketch of Lorentz's derivation. Poincare simply used the Lorentz transformations from an ether-rest frame $S$ to $S^{\prime}$, which in their $d x$ and $d t$ form are $d x^{\prime}=\gamma d(x-v t)$ and $d t^{\prime}=\gamma d(t-v x)$, in that way he got $u_{x}^{\prime}=\frac{d x^{\prime}}{d t^{\prime}}=\frac{\gamma d(x-t)}{\gamma d(t-v x)}=\frac{d x-v d t}{d t-v d x}=\frac{u_{x}-v}{1-v u_{x}}$. For brevity and simplicity I only refer to the $x$-velocity transformation. Poincare corrected the remaining velocity transformations and the charge density transformations by means of the same maneuver.

18 'Symmetric' could be a confusing term here, it is used with a different sense in contemporary theoretical physics, for example. However, I prefer it to 'reciprocal' because 'symmetric' is the term that is normally used in the literature. The
} 
that the coordinate transformations become $x^{\prime}=\gamma l(x+v t), y^{\prime}=l y, z^{\prime}=l z$, and $t^{\prime}=\gamma l(t+v x)$; that is, $-v$ is substituted by $+v$. Poincaré showed that for the inverse transformations $x^{\prime}=\frac{\gamma}{l}(x+v t), y^{\prime}=\frac{y}{l}$, $z^{\prime}=\frac{z}{l}$, and $t^{\prime}=\frac{\gamma}{l}(t+v x)$ to be part of the group, the factor $l$ must be set to 1 (Poincaré also showed that 1 is the only value for $l$ that excludes the possibility of detecting motion with respect to the ether). It is clear that with $l=1$ the inverse transformations are identical to the $y$-rotation case, and if this is so, the Lorentztransformations are symmetrical.

A physical consequence of this symmetry is that the length-contraction effect becomes also symmetric. If rod $B$ that moves in the ether-rest frame $S$ gets contracted with respect to the rod $A$ at rest in $S$, in a frame $S^{\prime}$ that moves with respect to the ether and in which $B$ is at rest and $A$ in motion, $A$ gets contracted with respect to $B$-we assume that when at rest with respect to each other $A$ and $B$ have the same length. This is also the case in special relativity, so the symmetry involved in the group-proof that Poincaré obtained is yet another crucial amendment to make the theories predictively equivalent. However, the explanations for this symmetry that the theories provide are different. In Einstein's it is just a consequence of the framedependence of simultaneity and the resultant frame-dependence of length ${ }^{19}$. In Lorentz's theory the rod $B$ gets really contracted, whereas the contraction of $A$ measured in the frame $S^{\prime}$ is a consequence of the 'wrong' synchronization of clocks in it. That is, since the clocks in $S^{\prime}$ measure the 'local time', the simultaneous events that determine the length of $A$ in that frame are not really simultaneous, and then the length measured is not the real one ${ }^{20}$.

The symmetry of the coordinate-transformations in the ether theory entails two non-empirical features that can be invoked to argue special relativity's superiority. First, it involves one of the asymmetries that do not belong to the phenomena that Einstein deprecated in the introduction of his 1905 paper. The explanation for the same physical effect, length-contraction, is different whether we consider the frame as at rest or as moving with respect to the ether. Second, the symmetry of the transformations entails that the velocity-withrespect-to-the-ether is not necessary for the derivation of length-contraction, clock-retardation or the velocity-dependence of inertial mass. The velocity involved is simply the relative velocity between the frames involved - this is clear if we consider the transformations from $S^{\prime}$ to $S$ in the previous example, in that case $v$ is the velocity of the ether-rest frame $S$ with respect to $S^{\prime}$, not the velocity of $S$ with respect to the ether, of course. This makes the ether not only undetectable, but also empirically superfluous.

One final contribution to the ether theory that Poincaré made in 1906 was that he noticed that the Lorentz transformations leave the quantity $c t^{2}-x^{2}+y^{2}+z^{2}$ invariant. Moreover, he noted that by defining a four-dimensional space with axes $x, y, z$ and $i t$, the transformations represent rotations of such a

property at issue is simply that the inverse transformation of a Lorentz transformation has the exact same form but substituting $+v$ for $-v$. Both Miller (1973) and Janssen (1995) use 'symmetric' to denote this property.

${ }^{19}$ That is, in special relativity the length of a rod is not an 'intrinsic' property of the object, it is the distance between the simultaneous events that coincide with the endpoints of the rod. Thus, if simultaneity is frame-relative, it follows that length is also so.

${ }^{20}$ See (Dorling 1968). For the observer in the system $S^{\prime}$ to be able to measure the real length of the $\operatorname{rod} A$, the reading of the clocks in $S^{\prime}$ should be corrected. In order to do so, the velocity of $S^{\prime}$ with respect to the ether must be known, but this is impossible given the theorem of corresponding states and the generalized contraction hypothesis. If the observer uses the method that Poincaré considers she will inexorably measure the 'local time'. 
space around a fixed origin, and he also discovered that some physical quantities, e.g. electric charge and current density, can be combined in four-component, Lorentz-invariant quantities. That is, Poincaré anticipated the seminal work of Herman Minkowski on the four-dimensional formulation of special relativity. However, unlike relativity in four-dimensional space-time, in the ether theory these properties represent mere mathematical niceties that do not have a physical meaning. As we will see below, Poincaré's glimpse at four-dimensional space-time is a relevant feature to assess one of the reasons that have been typically invoked to favor Einstein's theory over Lorentz's, namely, mathematical elegance.

After this outline of the development of Lorentz's theory, we can clearly see that the theory which is empirically equivalent to special relativity is not the one that Lorentz formulated in 1904. The amendments and contributions that Poincaré introduced in his 1906 work and the observations and criticisms that he leveled ca. 1900 must be considered to really obtain the predictive equivalence. In other words, the case of empirical equivalence at issue concerns Einstein's theory and a conceptual reconstruction that we could call the Lorentz-Poincaré theory. In what follows, when I speak of Lorentz's theory it is this conceptual reconstruction what I am referring to.

\section{Einstein vs. Lorentz in the light of non-empirical features}

I will review four theoretical, non-empirical features in the theories involved that could be used as arguments to make a choice favoring special relativity. First, I will consider the ad-hocness accusations leveled against the Lorentz-Fitzgerald contraction hypothesis; if this hypothesis is proved to be ad hoc, the epistemically problematic status of ad hoc hypotheses could thus ground a rejection of Lorentz's theory. Second, aesthetic features such as mathematical simplicity and elegance in special relativity have been used as a reason to favor it over Lorentz's theory. The work of Hermann Minkowski is crucial in this case, for the chrono-geometric formulation of special relativity in terms of Minkowski space-time is supposed to spell out its mathematical-aesthetic superiority over its rival. Third, the explanatory power of special relativity is also a candidate to ground a choice. This argument has been recently introduced and developed by Michel Janssen: this author claims that the fact that the Lorentz-invariance of all the laws of physics cannot be explained in Lorentz's theory-whereas in Einstein's such invariance is naturally explained by the metric of Minkowski space-time - determines the explanatory superiority of special relativity. Finally, I will propose yet another possible reason that (to my knowledge) has not been considered in the literature. I will show that once Poincaré established the symmetry of the coordinate transformations in Lorentz's theory, the ether postulated by the theory becomes not only undetectable, but also superfluous. I will consider each of these arguments in turn.

\subsection{The Lorentz-Fitzgerald contraction and ad-hocness}

In The Logic of Scientific Discovery, Karl Popper offered a definition of ad hoc hypotheses within the context of his falsificationist framework. A hypothesis is called ad hoc if it is unfalsifiable, that is, if the 
hypothesis does not entail any predictions that could put it 'at risk'. He mentioned the Lorentz-Fitzgerald length-contraction as a paradigmatic example of an ad hoc hypothesis - and this judgment became very influential regarding the epistemological assessment of Lorentz's theory. As we saw above, the LorentzFitzgerald contraction was originally introduced with the specific goal of providing an account for the negative result of the Michelson-Morley experiment. If this were the only empirical prediction for which the hypothesis is logically relevant, then it would clearly qualify as ad hoc in Popper's sense. Since Popper stated as a methodological principle that the introduction of new hypotheses in a given theory is allowed only if such hypotheses increase the degree of falsifiability of the theory, then the ad-hocness of lengthcontraction is reason enough, according to Popper, to dismiss Lorentz's theory and favor special relativity:

An example of an unsatisfactory auxiliary hypothesis would be the contraction hypothesis of Fitzgerald and Lorentz which had no falsifiable consequences but merely served to restore the agreement between theory and experiment - mainly the findings of Michelson and Morley. An advance was here achieved only by the theory of relativity which predicted new consequences, new physical effects, and thereby opened up new possibilities for testing, and for falsifying, the theory. (Popper 2002, pp. 62-3).

Adolf Grünbaum (1959) showed that this view was mistaken. The Kennedy-Thorndike experiment does provide a potentially falsifying prediction if the Lorentz-Fitzgerald hypothesis is assumed. The difference between the Kennedy-Thorndike and the Michelson-Morley experiment is simply that the two arms of the interferometer have different lengths, $L$ and $l$. Grünbaum showed that both assuming and not assuming the length-contraction hypothesis, the expected outcome is a difference in the travel time for light rays along the different paths in the interferometer. That is, in both cases a positive result, testifying the effect of the motion of the earth through the ether, was expected. Grünbaum's main point is that the value for the difference in travel time is given by $\frac{2}{\sqrt{v^{2}-c^{2}}}(L-l)$ if length-contraction is assumed; but if it is not, the time-difference is given by $\frac{2}{\sqrt{v^{2}-c^{2}}}\left(L-\frac{l}{\sqrt{1-v^{2} / c^{2}}}\right)$. In other words, in the case of the Kennedy-Thorndike experiment, the Lorentz-Fitzgerald contraction hypothesis did entail a risky prediction.

Popper acknowledged this point, but he did not change his conclusion. He simply adjusted his view in terms of degrees of ad-hocness and concluded that Lorentz's theory was more ad hoc than Einstein's:

Professor Grünbaum's correction shows that this hypothesis was testable and thus not ad hoc to the degree I believed. Accordingly it was an advance. But it was, of course, more ad hoc than special relativity. In other words, we have here an excellent example of 'degrees of ad hocness' and of one of the main theses of my book - that degrees of ad hocness are related (inversely) to degrees of testability and significance. (Popper 1959, p. 50).

But this whole line of reasoning is misconceived. It settles the issue by considering the lengthcontraction hypothesis in isolation with respect to the theory it belongs to. As mentioned above, the risky prediction that Grünbaum refers to is a positive result for the Kennedy-Thorndike experiment, but it turns out that one of the explicit goals of Lorentz's theory was to provide an explanation for the negative results obtained in all optical experiments to measure the effects of the earth's velocity with respect to the ether. 
That is, within Lorentz's theory the contraction hypothesis must entail a negative outcome for the KennedyThorndike experiment — otherwise its observed result would remain unexplained. Such a prediction can be obtained only if the 'clock-retardation' effect is also considered, and this effect, in turns, results from the concept of 'local time'"21.

Grünbaum was aware of this point ${ }^{22}$, but he simply concluded that 'only a version of the aether theory incorporating both the Lorentz-Fitzgerald contraction and the Lorentz-Larmor-Poincaré time dilation is vulnerable to the ad hoc charge, which tradition and Professor Popper have unjustly leveled against the first of these two auxiliary hypotheses alone' (Grünbaum 1959, p. 50). But if it is the ether theory including both the hypotheses (length-contraction and clock-retardation) what is subject to the ad hoc accusation, then the choice favoring special relativity holds, in spite of Grünbaum's argument.

It is not difficult, though, to demonstrate that the length-contraction hypothesis is not ad hoc in Popper's sense. As we saw above, in the 1895 version of Lorentz's theory the contraction hypothesis was indeed disconnected from the rest of the theoretical core of the theory. Thus, it was relevant only for the explanation of the negative result of the Michelson-Morley experiment, or, at best, for the explanation of the negative results of experiments of the same kind, as Grünbaum showed. Nevertheless, as also mentioned above, in the 1899 and 1904 versions of the theory the length-contraction hypothesis was fully connected to the theorem of corresponding states via the generalized contraction hypothesis. In the definitive formulation of the theory, the length-contraction hypothesis gets generalized and connected to the theorem of corresponding states in the form of the generalized contraction hypothesis - that is, the length-contraction hypothesis becomes a special case of the latter hypothesis. In turn, the theorem of corresponding states and the generalized contraction hypothesis are responsible for the derivation of the velocity-dependence of mass. Therefore, the hypothesis of length-contraction — which in the 1895 version of the theory could indeed be accused of ad-hocness in Popper's sense - becomes testable and falsifiable in a substantial way in the definitive version of Lorentz's theory. Therefore, it is not justified to invoke ad-hocness of the lengthcontraction hypothesis in order to dismiss Lorentz's theory ${ }^{23}$.

\footnotetext{
${ }^{21}$ In the 1895 version of the theory, the coordinate transformations that included the auxiliary quantity of 'local time' and the length-contraction hypothesis were two disconnected parts of the theory, so that in that version, a negative result for the Kennedy-Thorndike experiment was not possible. However, with the exact 1899 version of the theorem of corresponding states, in connection with the generalized contraction hypothesis, the clock-retardation effect required to explain the negative result of the Kennedy-Thorndike got interlocked with the length-contraction effect.

22 'Lorentz himself was quite clear that the contraction hypothesis would not suffice to make the body of experimental findings known at the time conform to the expectations of the aether theory. He therefore invoked the further auxiliary hypothesis known as the Lorentz-Larmor-Poincaré time dilation, construed as issuing a spurious 'local' time in all systems moving relatively to the ether' (Grünbaum 1959, p. 50).

${ }^{23}$ As Michel Janssen puts it: 'to examine the testability of a hypothesis, we also need to examine the theory in which the hypothesis is embedded, in this case, the version of Lorentz's theory based on the first-order theorem of corresponding states. This theory was set up to deal with first-order experiments. The contraction hypothesis was added to deal with one particular second-order experiment. The proposed contraction, a second-order effect, in principle can affect a wide range of phenomena, providing an equally wide range of conceivable tests. The problem is that a first-order theory cannot be used to predict the consequences of second-order effects. Thus, the most we can expect of the theory is that it will give definite predictions for the outcome of experiments that are but minor variants of the Michelson-Morley experiment, such as the Kennedy-Thorndike experiment. The possibilities of testing the contraction hypothesis in its original form are thus very limited. This is not true of the generalized contraction hypothesis, which is part of Lorentz's mature theory based on the exact theorem of corresponding states. This theory gives definite predictions for any experiment used to test it. There is, of course, something peculiar about these predictions. The prediction is always that no effect of the earth's presumed motion through the ether will be detected.
} 
Besides Popper's, though, there remain two senses in which the length-contraction hypothesis might be accused of ad-hocness. First, it was cooked up with the only and specific goal of solving one single experimental difficulty. This is true, but as we just saw, further development of the theory connected the hypothesis with unexpected empirical results. In this sense, the length-contraction hypothesis is on an analogous stand with Planck's quantum of energy hypothesis - which was introduced with the only and specific goal of providing an account for the observed spectrum of black-body radiation — and in neither of the two cases this sense of ad-hocness could be invoked to reject the corresponding hypothesis, of course. Moreover, one could ask what is wrong with ad hoc hypotheses-in the sense of cooked up hypotheses-in themselves. Even if a hypothesis is helpful in providing an explanation for one single experimental result, it does contribute to enlarge the scope and fruitfulness of the corresponding theor ${ }^{24}$.

The second remaining sense of ad-hocness that could be attributed to the length-contraction hypothesis is that it was a sort of rabbit in the hat maneuver, that is, that the contraction was postulated without a justified physical underpinning. In this case, the ad-hocness accusation boils down to an accusation of implausibility. We saw above that Lorentz did propose a plausibility argument for his length-contraction hypothesis, and the fact that the same argument had been already envisioned by Fitzgerald bestows Lorentz's with good credentials. Moreover, the eventual fulfillment of the main goal of the electromagnetic worldview program — namely, the reduction of all physics to electromagnetism—would have provided the generalized contraction hypothesis with firm physical foundations.

However, as we saw above, in 1906 Poincaré made clear that the required corrections in Lorentz's model of the electron included the postulation of a non-electromagnetic quantity, Poincaré-pressure. This meant that the goal of the program of reducing all physical laws to electrodynamics was not fulfilled. This episode in the historical development of Lorentz's theory has been interpreted by Kenneth Schaffner (1974) as opening a flank for an ad-hocness accusation of what he calls the 'molecular forces hypothesis' (which pretty much corresponds to Janssen's generalized contraction hypothesis):

This is a problematic feature of the theory. But it has nothing to do with lack of testability. All that matter on that score is that the theory predicts a definite result. The generalized contraction hypothesis therefore passes Popper's falsifiability test with flying colors, whereas the original contraction hypothesis earned only a marginally passing grade courtesy of Roy Kennedy and Edward Thorndike' (Janssen 2002a, p. 433). The prediction of the velocity-dependence of mass for which the generalized contraction hypothesis is relevant counts as an exception of the 'negative-results' drawback that Janssen mentions.

${ }^{24}$ As Larry Laudan states it: ' $a$ theory is ad hoc if it is believed to figure essentially in the solution of all and only those empirical problems which were solved by, or refuting instances for an earlier theory [...]

Assuming that adhocness is understood in this way, we are entitled to ask: what is objectionable about it? If some theory $T_{2}$ has solved more empirical problems than its predecessor-even just one more - then $T_{2}$ is clearly preferable to $T_{1}$, and, ceteris paribus, represents cognitive progress with respect to $T_{1}$. [..]

In urging that adhocness (so defined) is a cognitive virtue rather than a vice, I am clearly not implying that ad hoc theories are invariably better than non-ad hoc ones. My claim, rather, is that an ad hoc theory is preferable to its non-ad hoc predecessor (which was confronted with known anomalies). [...]

But it might be argued that I have missed the point of the critics of adhocness. They might say yes, of course, $T_{2}$ is better than its refuted predecessor $T_{1}$; but the relevant comparison is between $T_{2}$ and some other theory $T_{n}$ which is not ad hoc but still solves as many problems as $T_{2}$ '. Einstein's special theory of relativity might exemplify $T_{n}$ while the Lorentz modified aether theory was $T_{2}$. The obvious reply to such criticism is to ask why the admittedly ad hoc character of the Lorentz contraction constitutes a decisive handicap against it comparing it with special relativity. If the empirical problem-solving capacities of the two theories are, so far as we can tell, equivalent, then they are (empirically) on a par; defenders of the view that the adhocness of $T_{2}$ makes it distinctly inferior to $T_{n}$ must spell out why, in such cases, the comparable problem-solving abilities and equivalent degree of empirical support can be thrown to the winds simply by stipulating that ad hoc theories are intrinsically otiose' (Laudan 1977, pp. 115-17). See also (Grünbaum 1976, section 4). 
There were serious reservations about the satisfactory applicability of such a generalized M.F.H. to electrons. [...] it was shown by Poincaré (1906) that the contractile electron could be considered a stable entity only if a definitively non-electromagnetic counter-pressure were invoked. To extend the M.F.H. to cover this type of force would violate the reduction thesis (of the M.F.H to electromagnetic forces) which provided the plausibility (and independent support) for the original M.F.H. (Schaffner 1974, p. 52).

These serious reservations, Schaffner argues, turned into an insurmountable problem with the advent of quantum physics. The conflict between electrodynamics and the quantum hypothesis was so deep that it became impossible to offer a physical-plausibility argument for the M.F.H.:

\begin{abstract}
If we examine the changing background assumptions of this time [early 1900s] I believe that we should see the weak rationale for some of Lorentz's hypotheses becoming even weaker. Consider the generalized M.F.H. discussed earlier. This hypothesis is ad hoc but it might be said to possess a slight degree of theoretical support on the supposition that Poincare's arguments concerning the non-electromagnetic nature of the force holding the electron together were misguided. If all forces were electromagnetic the generalization of the M.F.H. would not be ad hoc but might follow from a deeper classical theory. The calling into serious question of the fundamental assumptions of the Lorentz electron theory by light quanta made such ad hoc assumptions seriously ad hoc, by introducing in advance, counterarguments to any such deeper classical theory. (Ibid., p. 75).
\end{abstract}

At this point, I will make only two brief comments about Schaffner's view. First, the problems with the M.F.H. do involve a non-empirical criterion that can be invoked to argue special relativity's superiority. However, issues concerning implausibility of theoretical features are better assessed in an explanatory context rather than in terms of accusations of $a d$-hocness ${ }^{25}$. This is the standpoint that Michel Janssen takes on this matter. He has argued that the failure of the electromagnetic program implies that a central feature of Lorentz's theory - the Lorentz-invariance of all dynamical laws, electromagnetic and non-electromagneticremains an unexplained coincidence. I will undertake a deep assessment of Janssen's position in section 3.3.

Second, the way in which Schaffner evaluates the relevance of the quantum hypothesis in the case of Lorentz vs. Einstein is, I think, misguided. It is true that the conflict between electrodynamics and quantum physics was a crucial factor in the historical course of events, and also that it provides a conceptually justified ground to definitively favor Einstein's theory. However, rather than in terms of ad-hocness (or of explanatory issues), the mentioned conflict gets better described in terms of non-consequential empirical evidence. I will develop this view in section 5.1.

\title{
3.2 Mathematic-aesthetic features
}

A second non-empirical feature that could be used in order to make a choice favoring Einstein's theory is given by the aesthetic-mathematical virtues that characterize special relativity. Actually, these features did

\footnotetext{
${ }^{25}$ As Schaffner points out, his sense of ad-hocness corresponds to Elie Zahar's concept of ad hoc . A theory is ad hoc $_{3}$ 'if it is obtained from its predecessor through a modification of the auxiliary hypotheses which does not accord with the spirit of the heuristic of the programme' (Zahar 1973, p. 101). In our case, the new hypothesis which violates the spirit of the original program is, of course, the M.F.H., and the accusation holds if it can be proven that there is no possible plausibility argument for the mentioned hypothesis in the context of the electromagnetic worldview program. Since the concept of ad hoc boils down to a matter of plausibility, I think that accusations of ad-hocness in this sense get better described in terms of the explanatory features of the theory involved.
} 
play a historically relevant role in the matter ${ }^{26}$. Consider, for example, the following passage included in Max von Laue's 1911 textbook (the first ever published) on special relativity:

Though a true experimental decision between the theory of Lorentz and the theory of relativity is indeed not to be gained, and that the former, in spite of this, has receded into the background, is chiefly due to the fact that, close as it comes to the theory of relativity, it still lacks the great simple universal principle, the possession of which lends the theory of relativity an imposing appearance. (Quoted in Schaffner 1974, p, 74)

Minkowski's introduction in 1908 of the four-dimensional formalism in which the theory can be expressed was a crucial factor regarding the general judgment about the mathematical simplicity of special relativity. Scott Walter (2010) has offered a historical survey of the early reception of Minkowski's work in connection with the acceptance of special relativity. He shows that Laue's specific reasons to embrace Einstein's theory in terms of simplicity was given — in spite of its difficult visualizibility in intuitive termsby the mathematical elegance and simplicity that the four-dimensional formulation allowed:

Laue considered Minkowski space-time as an "almost indispensable resource" for precise mathematical operations in relativity. He expressed reservations, however, about Minkowski's philosophy, in that the geometrical interpretation (or "analogy") of the Lorentz transformation called upon a space of four dimensions: "[A] geometric analogy can exist only in a four-dimensional manifold. That this is inaccessible to our intuition should not frighten us; it deals only with the symbolic presentation of certain analytical relationships between four variables". One could avail oneself of the new four-dimensional formalism, Laue assured his readers, even if one was not blessed with Minkowski's space-time intuition, and without committing oneself to the existence of Minkowski's four-dimensional world' (Walter 2010, p. 17). ${ }^{27}$

But what are the precise aesthetic virtues that the four-dimensional presentation of the theory contains? In what sense mathematical simplicity is being considered? Peter Galison (1979) has offered a detailed account that we can use as a sample. He states that the virtues are three: symmetry, generality and invariance. The symmetry that Galison refers to is geometric. In Minkowski space-time, provided that an arbitrary event is assigned with the coordinates $(0,0,0,0)$, the set of all possible space-time coordinates for one event is given by the four-dimensional hyperboloid $c t^{2}-x^{2}-y^{2}-z^{2}=C$. The Lorentz transformations allow that any point on the hyperboloid can be transformed to lie on the $t$-axis - and for the corresponding vector, velocity equals zero. Galison's point is that the Lorentz transformation applied to a specific event-point takes

\footnotetext{
${ }^{26}$ See (Brush 1999).

${ }^{27}$ As Walter points out, Emil Wichert took a similar stance: 'Another physicist, Minkowski's former colleague and director of the Göttingen Institute for Geophysics, Emil Wiechert welcomed Minkowski’s space-time theory, but felt there was no need to dismiss absolute space. Following a remark made by Minkowski in "Raum und Zeit," Wiechert proposed to recover the notion of direction in Euclidean space with what he called "Schreitung" in space-time, or what amounted to the direction of a four-velocity vector. As for Minkowski's claim that a new intuition of space and time was required, this did not bother Wiechert at all. In a non-technical review of relativity theory, Wiechert wrote that the special relativity theory was "brought by Minkowski to a highly mathematically-finished form." He continued: "It was also Minkowski who, with bold courage, drew the extreme consequences of the theory for a new space-time-intuition [...] and contributed so very much to the theory's renown". It was precisely Minkowski's space-time-intuition, or his identification of the extreme consequences of this intuition, that had made the theory of relativity famous in Wiechert's view. For Wiechert, however, all intuitions, including ether, and matter in motion, were but anthropomorphic "images," the reality of which was beyond our ken' (Walter 2010 , p. 16).
} 
the hyperboloid back into itself — and the physical consequence is that absolute (ether) rest becomes undefined $^{28}$.

The generality that Galison explains consists in that the four-dimensional geometry that the German mathematician introduced allows us to express different groups of invariant transformationstransformations which in turn determine different space-times with different metrics. The transformations in which the velocity of light takes the constant and frame-independent value of $c$ correspond to the group of Lorentz transformations $G_{C}$ and express the metric of Minkowski space-time. If the transformations do not pose an upper limit to the velocity of light, the corresponding group is $G_{\infty}$, the Galilean transformations that determine a space-time with Newtonian metric.

Finally, the third aesthetic factor that Galison points out is invariance:

The existence of invariants for the relativistic transformations forms the third aesthetic criterion Minkowski considers in his four-dimensional relativistic theory. "The innermost harmony of these [electrodyamic] equations", he writes, "is their invariance under the transformations of the expression $d x^{2}+d y^{2}+d z^{2}-$ $d t^{2}$ into itself'. In Newtonian space-time the free $t$-axis prevents us from constructing such an invariant expression. Like symmetry and generality, invariance is an aesthetic geometric-criterion which supports the new conception of space-time. (Galison 1979, pp. 111-2)

Mathematical-aesthetic features like these could thus be invoked in order to decide the contend between special relativity and Lorentz's theory. It is true that aesthetic features are problematic from an epistemological point of view. Questions like 'why is beauty desirable in scientific theories?' or 'can a theory be objectively evaluated as more beautiful than a rival?' are open philosophical problems. However, even if we take for granted that aesthetic features can be objectively assessed and that they can ground theory-choice in a justified way, in the case of Lorentz vs. Einstein they cannot be invoked anyway.

Galison's mathematical-aesthetic features are clearly rooted in the four-dimensional mathematical structure that Minkowski introduced, and the quotes above from Laue are consistent with this view. We saw that in 1906 Poincaré derived some mathematical results of Lorentz's theory. He noticed that the expression $c t^{2}-\mathbf{x}^{2}$ is invariant under Lorentz transformations, so that the transformations can be understood not only as rotations around the $x$ and $z$ axes, but also around the $x$-axis and a fourth axis ict. That is, Poincaré showed that Lorentz's theory can be formulated in the four-dimensional geometric language that Minkoswki developed in 1908. Therefore, all the mathematical-aesthetic virtues that can be predicated of special relativity in terms of its formulation in a four-dimensional geometry can also be assigned to Lorentz's theory.

\footnotetext{
28 'Different observers assign different coordinates to a given event. Minkowski reasons that since $t^{2}-x^{2}-y^{2}-z^{2}$ is Lorentz-invariant, the four-dimensional hyperboloid $t^{2}-x^{2}-y^{2}-z^{2}=$ constant represents the set of all possible spacetime coordinates of one event. The principle of relativity tells us that "absolute rest corresponds to no properties of the phenomena". Since in four dimensions there is a non-zero vector lying on the hyperboloid and corresponding to zero velocity, any point $(x, y, z, t)$ on the hyperboloid can be transformed to lie on the $t$-axis. Such a Lorentz transformation will take the hyperboloid back into itself. This is the geometric symmetry which Minkowski introduces into relativity. Its physical consequence is that no particular measurement of the coordinates of an event can indicate absolute rest. [...] The four-dimensional representation places rest and motion on equal graphical footing. Since any four-vector can be transformed to the "rest-vector", leaving the hyperboloid of the appropriate invariance unchanged, the principle of relativity, i.e., that no phenomena are attached to absolute rest, stands fully exposed. Such a symmetry is clearly distinct from the physical symmetry of Einstein [Galison here refers to Einstein's motto 'theoretical asymmetries that do not reflect in the phenomena'] and the formal group or group symmetries of Poincare' (Galison 1979, pp. 104-5).
} 
According to Einstein's theory, the Lorentz transformations express the Minkowskian metric of space-time, so that its formulation in terms of a four-dimensional geometry has to be understood in a 'literal' physical way. On the other hand, since Lorentz's theory does not depict a Minkowski space-time, but a Newtonian one, the fact that the theory can be formulated in a four-dimensional geometric language can only be understood as a mathematical nicety that does not express its physical content. However, this is enough in order to state that Lorentz's theory can be presented in a way such that the mathematical virtues that are assigned to special relativity can also be assigned to it. Therefore, mathematical-aesthetic features cannot be used in order to make a choice in this case ${ }^{29}$.

So far we have seen that two of the non-empirical features that have been commonly referred to in order to settle a decision in the Einstein vs. Lorentz case are not justified from a conceptual point of view. In the next two sections I will deal with two further non-empirical features which are more promising.

\subsection{Janssen's argument}

Michel Janssen has offered a very influential argument regarding the case of Lorentz vs. Einstein (1995, 2002a, 2002b, 2009, and Balashov and Janssen 2003). This argument relies on the different way in which the theories involved draw the line between dynamics and kinematics. Recall that in Lorentz's theory effects like clock-retardation, length-contraction and the velocity-dependence of mass are grounded on a peculiarity of the laws that govern the microstructure of all physical systems, namely, their Lorentz-invariance. Thus, the Lorentz-invariance of all physical laws has a dynamical foundation within Lorentz's theory. The ultimate dynamical ground of such invariance could be traced to the interaction between electromagnetic systems and the ether. Therefore, for the supporters of the electromagnetic worldview, Lorentz-invariance was a natural feature of physical laws. In the case of special relativity, the Lorentz-invariance of the laws of physics is not dynamically grounded. The mentioned physical effects are a manifestation of the new and revolutionary kinematics that the theory postulates. This feature of the theory became clear and explicit in the seminal work of Minkwoski: the metrical features of Minkwoski space-time- -which govern the normal spatiotemporal behavior of physical objects - determine that the laws governing physical systems are Lorentzinvariant. That is, in special relativity the Lorentz-invariance of physical has a kinematical, chronogeometrical foundation.

\footnotetext{
${ }^{29}$ My argument is not analogous to say that a genuinely virtuous person is no better than a thief that dresses just as the virtuous people do. My point is that, in and by itself, the clothes of the virtuous and the thief cannot make a difference with respect to their moral assessment — what makes them virtuous or vicious is not their clothes, they both have the same wardrobe. It might be argued that in the case of the virtuous, his choice of clothes is somehow connected to his morals (he might use the same mental faculty to choose his clothes and to determine his behavior), but that cannot be argued only in terms of the aesthetic features of his outfit. Something analogous occurs in the case of special relativity and Lorentz's theory. The mathematical features of the former reflect physical features. For example, Galison's symmetry, insofar as it is connected to the impossibility of defining rest in an absolute sense, is not a merely mathematical feature of special relativity, but it is linked to the physical content of the theory. Actually, it cannot be strictly applied to Lorentz's theory, for in it rest with respect to the ether is qualitatively distinct to relative rest (though the symmetry that Galison points out could be taken as reflecting the fact that the state of absolute (ether) rest is empirically undetectable in Lorentz's theory). Therefore, Galison's concept of symmetry manifests the physical simplicity of special relativity. However, this does not mean that the theories at stake do not stand on an equal foot regarding (strictly) mathematical virtues connected to their four-dimensional formulations. Physical simplicity is not only a matter of aesthetics. As I will argue below, in the case of special relativity vs. Lorentz's theory, this feature manifests the firmer physical foundations of the former.
} 
Janssen argues that special relativity can be preferred over Lorentz's theory because in the former the kinematical explanation of Lorentz-invariance has the form of a common origin inference (COI). In Lorentz's theory, there is no common origin to explain the fact that all the laws of physics are Lorentzinvariant. Lorentz developed his theory from the fact that electromagnetic laws are Lorentz-invariant-recall his plausibility argument for the length-contraction. However, the mature version of his theory required that all the laws of physics have the property at issue. If a unified explanation of Lorentz-invariance is not available, then the fact that both electromagnetic and non-electromagnetic laws of nature possess that property becomes a sort of cosmic coincidence. There was a time when the electromagnetic worldview, that intended to reduce all physics to electrodynamics, offered a promising possibility: if Lorentz-invariance is characteristic of electromagnetic laws, and if all laws are electromagnetic, then the Lorentz-invariance of all laws is guaranteed and explained by a common origin. However, the necessary introduction of Poincarépressure implied that a full reduction of physics to electrodynamics was not achieved ${ }^{30}$.

This argument, of course, requires further epistemological justification. After all, a similar unexplained coincidence in Newton's theory - the equivalence between gravitational and inertial mass - did not challenge its acceptance. It is true that Newton's theory was replaced by general relativity, and a salient feature of the latter is that contains a common origin explanation for the mentioned equivalence. However, general relativity defeated Newton's theory on the empirical-evidence battleground. In a case of empirical equivalence, such as special relativity vs. Lorentz's ether theory, it must be shown that a choice based on a specific $C O I$ argument is epistemologically justified. That is, it must be explained why to have a $C O I$ explanation is better than not having it.

Concerning such justification, Janssen has developed and subtly modified his argument along time. The first version of the argument (1995, 2002a, 2002b) is of a causal nature, Minkwoski space-time is taken as the cause of Lorentz-invariance:

The contraction of physical systems and the retardation of processes in such systems when the system is set in motion, no matter whether the system is of an electromagnetic or of a non-electromagnetic nature, are effects that seem to have a common cause in special relativity, but that are due to unexplained coincidences in the ether theory. (Janssen 1995, section 4.2.1)

Lorentz invariance manifests itself in many different phenomena. Ultimately, these phenomena form the input of the common-cause argument. The most obvious examples are length-contraction and time dilation. The length of a system in uniform motion is less than that of an identical system at rest by a factor that depends only on the velocity of the moving system. A process in the moving system takes longer than the corresponding process in the system at rest by that same factor. In the Newtonian space-time of Lorentz's theory this is a consequence of the unexplained coincidence that all of these systems are governed by Lorentz-invariant laws. In the Minkowski space-time of special relativity, it is a consequence of the way in which particular space-time slices are used to define the length of a system or the duration of a process. (Janssen 2002a, p. 439).

\footnotetext{
${ }^{30}$ At this point we can see a similarity between Janssen's and Schaffner's arguments. Schaffner states that the M.F.H. became definitively ad hoc $_{3}$ with the rise of quantum physics. That is, no possible explanation for the physical underpinning of the Lorentz-invariance of non-electromagnetic dynamical laws could be offered - whereas special relativity was not ad hoc in this sense. Janssen claims that the Lorentz-invariance of all physical remains an unexplained coincidence, whereas in special relativity this feature gets naturally explained by the kinematics of the space-time postulated by the theory.
} 
To speak of space-time as a common cause is of course a risky maneuver, for it seems to imply a substantivalist position concerning space-time - and the question of the ultimate ontology of space-time is an open, philosophical problem. This is why Janssen (1995) softened his position by stating that his argument is of a common cause type:

The reason for calling this a 'common cause'-type argument rather than a common cause argument, is that Minkowski space-time does not seem to be a common cause in quite the same sense that a shrimp cocktail contaminated with the salmonella bacteria is the common cause of the sudden death of half the population of a cheap Dutch old folks home. [...]

Although the status of the 'common cause' obviously needs further philosophical clarification, it is safe to say, I think, that this is a very strong argument for preferring special relativity over an empirically equivalent classical ether theory. (Janssen 1995, section 4.2.1).

Unlike Janssen (1995), I think that it is very unsafe to argue that space-time can be a cause of anything. This position, in spite of the softening clause, seems to be essentially committed to a substantivalist view, and the relationist would surely reply that there is no sense in which space-time can be a cause. That is, the proviso that the argument is of a common cause type is not enough to avoid the problematic commitment regarding the ontology of space-time.

In his (2002b), Janssen still argues for a causal argument. Minkowski space-time is again attributed a causal role in the sense that it is responsible for the Lorentz-invariance of physical laws. However, this time Janssen is explicit in that we should not take this as an indication that Minkowski space-time is a substance. To understand Minkowski space-time as a structure is enough in order to endow it with casual powers:

What makes the special-relativistic explanation of Lorentz invariance of physical laws a good explanation? Is it because it provides a unified account or because it provides a causal account? In other words, does the example support Salmon's causal account of explanation or Friedman and Kitcher's unification account? If unification were all that matters, it is unclear why Lorentz's theory does not provide a perfectly adequate explanation of length-contraction. Lorentz's claim that all laws are Lorentz invariant has tremendous unifying power. On the basis of this one claim it can account for everything special relativity can account for. Yet, the theory is unsatisfactory from an explanatory point of view. It does not have the resources to explain why physical systems in the Galilean space-time posited by the theory are all governed by Lorentz-invariant laws. In short, Lorentz's theory unifies but does not explain. [...]

At first glance, Salmon's causal account is unsatisfactory as well. Minkowski space-time certainly explains length-contraction, but it hardly qualifies as a causally efficacious substance. This objection can be avoided by broadening Salmon's concept of causation [...]. The COI in this case is to a structure rather than a substance. In this way Salmon gets this example right: special relativity explains and Lorentz's theory does not. (Janssen 2002b, p. 501).

I think that this maneuver does not do the trick either. It is not clear at all what does it mean that Minkowski space-time is a structure with causal powers. Is it a physical structure such as a crystal? This position would be very hard to defend. But if it is an abstract structure then it is totally unclear in what way such a structure can be the cause of anything. Harvey Brown makes a similar objection. When considering the argument that Minkowski space-time explains the Lorentz-invariance of physical laws he asks 'So how is 
its influence on these laws supposed to work? How in turn are rods and clocks supposed to know which space-time they are immersed in?'(Brown 2005, p. 143).

In a subsequent paper, Balashov and Janssen (2003) tried to disentangle the argument from any ontological presuppositions about space-time, while conserving the COI kinematic explanation for Lorentzinvariance that special relativity offers. They make their point by means of an analogy. They ask us to imagine Cyrano running off Roxanne's house. As Roxanne sees Cyrano, he turns and goes away. As he turns, Roxanne sees his nose to become more and more prominent, then to get smaller and smaller until it vanishes. Balashov and Janssen claim that it is the structure of Euclidean space what explains why the laws that hold Cyrano's nose together are invariant under rotations. They also claim that, analogously, it is the structure of Minkowski space-time what explains that physical laws are Lorentz-invariant. This way to present the matter still retains a substantivalist flavor. However, Balashov and Janssen then state that, even under a relationist ontology, the arrow of the explanation points in the same direction:

Since the invariant group (Lorentzian or Galilean) of the dynamical laws essentially is the space-time structure for a relationist, the (effective) Lorentz invariance of the dynamical laws in a sense does seem to explain for a relationist why the (effective) space-time structure is Minkowskian. Such an explanation, of course, does not amount to an explanation of why the space-time structure is Minkowskian rather than Newtonian [...]. Nor does it interchange the role of explanans and explanandum in our Cyrano-and-Roxanne example. The behavior of Cyrano's nose is just an instance of the normal spatio-temporal behavior of objects in Minkowski space-time, no matter whether one is a substantivalist or a relationist about the ontology of space(-time). The explanatory considerations in the text are therefore largely independent of one's stance on the ontology of space(-time). (Balashov and Janssen 2003, p. 341, footnote 11).

I think that in this passage Balashov and Janssen do approach a formulation of the explanatory nature of special relativity independent of ontological assumptions about space-time. The key sentence is that 'the behavior of Cyrano's nose is just an instance of the normal spatio-temporal behavior of objects in Minkowski space-time', meaning that the Lorentz-invariance of physical laws encodes the universal spatiotemporal behavior of physical objects. The clause 'objects in Minkowski space-time' still retains a substantivalist flavor, but we can understand it - and I think this is what Balashov and Janssen have in mind - as stating that the natural spatio-temporal behavior of physical objects is given by the kinematics encoded in the Lorentz-invariance of physical laws.

Janssen, though, interprets this manner of displaying the explanatory form of special relativity in a way I do not agree with. He claims that the fact that Minkowski space-time explains the Lorentz-invariance of physical laws entails that a constructive explanation of the invariance is given by special relativity ${ }^{31}$ :

\footnotetext{
${ }^{31}$ Einstein's famous distinction between 'theories of principle' and 'constructive theories' is precisely and succinctly explained by Balashov and Janssen: 'In a theory of principle, one starts from some general, well-confirmed empirical regularities that are raised to the status of postulates (e.g., the impossibility of perpetual motion of the first and second kind, which became the first and second laws of thermodynamics). With such a theory, one explains the phenomena by showing that they necessarily occur in a world in accordance with the postulates. Whereas theories of principle are about the phenomena, constructive theories aim to get at the underlying reality. In a constructive theory one proposes a (set of) model(s) for some part of physical reality (e.g., the kinetic theory modeling a gas as a swarm of tiny billiard balls bouncing around in a box). One explains the phenomena by showing that the theory provides a model that gives an empirically adequate description of the salient features of reality.' (Balashov and Janssen 2003, p. 331).
} 
For Einstein special relativity was a theory of principle. With the introduction of Minkowski space-time, however, it became a constructive theory. Minkowski space-time is the structure responsible for all the effects derivable from special relativity alone. Special relativity, from this point of view, replaced Newtonian space and time by Minkowski space-time and does not make any claims about the contents of the new spacetime other than their spatio-temporal behavior had better accord with Minkowski's new rules. (Janssen 2002b, p. 506).

Both the space-time and the Neo-Lorentzian interpretation [of special relativity] provide constructive-theory explanations. In the space-time interpretation, the model is Minkowski space-time and length-contraction is explained by showing that two observers who are in relative motion to one another and therefore different sets of space-time axes disagree about which cross-sections of the 'world-tube' of a physical system give the length of the system. (Balashov and Janssen 2003, p. 331).

This view becomes problematic insofar as the constructive nature of the explanation, according to Janssen, still holds in the case of its ontological commitments-free formulation. In his (2009), Janssen introduces important amendments and remarks that aim to detach his stance from any possible ontological presuppositions about space-time. For example, he states that his argument is no longer of a causal type:

I claim that Minkowski space-time explains Lorentz-invariance. For this to be a causal explanation, Minkowski space-time would have to be a substance with causal efficacy. Like Brown, I reject this view [...]. As I hope to make clear, Minkowski space-time explains by identifying the kinematical nature (rather than the cause) of the relevant phenomena.

Special relativity, as a physical theory, is agnostic about the ontology of space-time. (Janssen 2009, p. 28). ${ }^{32}$

After setting the epistemological framework of his argumentation, that is, after being explicit in that his position is completely independent of specific ontological stances regarding space-time, Janssen still argues that the kinematic explanation of Lorentz-invariance qualifies as a constructive one:

The Lorentz invariance that can be derived from the postulates (in conjunction with the assumption that space and time are homogeneous and isotropic) finds its natural interpretation in terms of the geometry of Minkowski space-time. On my definition of the principle-constructive distinction ${ }^{33}$, this interpretation amounts to the constructive-theory version of special relativity. It says that the space-time component of any acceptable model of a world in accordance with the postulates is Minkowski space-time. (Ibid, p. 39)

Minkowski (1909) did for special relativity, understood strictly as a principle theory, what Boltzmann had done for the second law of thermodynamics. It turned special relativity into a constructive theory by providing the concrete model for the reality behind the phenomena covered by the principle theory. (Ibid, p. $40)$.

I think that the problem with Janssen's position is quite clear. If, as Einstein's and Janssen's definition states, a constructive theory aims to unravel the underlying reality behind the phenomena, how is it possible that the Minkowskian formulation of special relativity can count as a constructive theory without in some

\footnotetext{
${ }^{32}$ Notice that these remarks strengthen the view that when Janssen uses the expression Minkowski space-time we have to understand something like 'the kinematics encoded in the Lorentz-invariance of physical laws'.

${ }^{33}$ For his definition of the principle-constructive distinction, Janssen remits to the definition presented in (Balashov and Janssen 2003) that I quoted in footnote 28.
} 
sense reifying Minkowski space-time? I think this is just impossible. The very definition of a constructive explanation precludes it $^{34}$.

After this review and critical assessment of Janssen's arguments, we can now consider the best possible formulation of what he regards as a reason to prefer special relativity. Recall that the basic idea is that in Lorentz's theory the fact that all the laws of physics are Lorentz-invariant remains as an unexplained coincidence; whereas in special relativity Lorentz-invariance gets naturally explained by the specific kinematics of space-time. For the reasons mentioned above, this explanation cannot be taken either as causal or as constructive. If it is an explanation at all, it has to be an explanation of principle.

A more formal presentation of this argument is offered in (Balashov and Janssen 2003, p. 341). This formulation relies on John Earman's symmetry principles: SP1) any dynamical symmetry of a theory $T$ is a space-time symmetry of $T$, and $S P 2$ ) any space-time symmetry of $T$ is a dynamical symmetry of $T$ (Earman, 1989, p. 46). In Lorentz's theory, the Galilean transformations express the spatio-temporal symmetries, but they do not express the symmetries of the dynamical laws. In turn, the Lorentz transformations express the symmetries of the dynamical laws, but they do not correspond to the spatio-temporal symmetries of the theory. This defect is not present in special relativity, of course. In this case the Lorentz transformations represent both the space-time and dynamical symmetries. In other words, Lorentz's theory violates Earman's principles, but special relativity respects them.

Janssen takes it that in Einstein's theory it is the space-time symmetries of the theory what explains the symmetries in the dynamical laws-I have argued that this explanation cannot be either causal or constructive (unless we endorse substantivalism). We are thus left with a principle-kinematic explanation. However, Janssen's stance - that the observance of Earman's principles in special relativity means that the (kinematic) space-time symmetries explain the dynamical symmetries - has been challenged by Harvey Brown and Oliver Pooley. They argue that the arrow of explanation points in the opposite direction: it is the dynamic symmetries what explains the space-time symmetries. Their argument to refute Janssen's view is that

as a matter of logic alone, if one postulates space-time structure as a self-standing, autonomous element in one's theory, it need have no constraining role on the form of the laws governing the rest of content of the

\footnotetext{
${ }^{34}$ Janssen's motivation for insisting in characterizing special relativity as a theory of principle in which Minkowski space-time plays the role of 'the reality underlying the phenomena' is that, concerning the comparative explanatory power of theories of principle and constructive theories, he endorses the following view: 'Brown and Pooley (2006, pp. 74-5) correctly point out that, contrary to what Balashov and I suggested, principle theories are not explanatory. Explanations are about the reality behind the phenomena (be it about their causes or about their nature). Principle theories, on the definition used by Balashov and me, are agnostic about that' (Janssen 2009, p. 38, footnote 27). However, I think that the situation is not that bad concerning principle theories and their explanatory power. To say that principle theories are not explanatory seems exaggerated. It makes more sense to argue that, in principle, and given a certain realm of phenomena, a constructive explanation is superior in terms of the understanding it provides of those phenomena when compared to an explanation of principle. The paradigmatic example of thermodynamics and statistical mechanics is illustrative. Complete understanding of the corresponding phenomena was given by means of the constructive theory, but that does not mean that the principle theory does not explain. Actually, in some contexts an explanation of principle might be superior to a constructive theory. Balashov and Janssen defend the explanatory power of principle theories referring to thermodynamics (2003, pp. 32-3), but Janssen abandoned this view in his (2009), as we just saw. Martin Frisch $(2005,2011)$ has offered an interesting comparison between Einstein's principle/constructive distinction and a very similar one introduced by Lorentz in 1900. He also argues that both Einstein and Lorentz (especially the latter) did assign an important explanatory value to the 'principle approach'.
} 
theory's models ${ }^{35}$. So how is its influence on these laws supposed to work? Unless this question is answered, space-time's Minkowskian structure cannot be taken to explain the Lorentz covariance of dynamical laws. (Brown and Pooley 2006, p. 84).

Brown and Pooley's point is that, as we saw above, the only way in which it can be argued that Minkowski space-time can constructively explain Lorentz-invariance of the dynamical laws is by reifying $\mathrm{it}^{36}$. But even if this step is taken, it is still unclear in what way the very peculiar entity space-time is can determine the behavior of physical objects ${ }^{37}$. The remaining alternative is not to postulate the space-time structure as autonomous and self-standing, but as determined by the peculiarities of the dynamical laws. But this view, Brown and Pooley argue, boils down to state that the arrow of explanation goes from dynamics to kinematics $^{38}$.

My intention here is not to take sides in the debate regarding which is the correct interpretation of special relativity, kinematical or dynamical, neither in the debate concerning the ontological status of space-time. My point is simply that if we follow Janssen's path in interpreting special relativity's compliance with Earman's symmetry principles in terms of a (kinematic) explanation of Lorentz-invariance, then we get involved in an epistemological, open dispute. The upshot would thus be that the reason we are invoking to decide the case of Einstein vs. Lorentz relies on shaky epistemological grounds. In other words, Janssen's argument to prefer special relativity over Lorentz's theory seems to presuppose that we are ready to follow him in that, in Einstein's theory, kinematics explains the dynamics.

We should then look for epistemic justifications of the symmetry principles that do not commit us to open epistemological problems. The justification that Earman himself proposes (1989, pp. 46-7) is useful in this context. He defends the symmetry principles grounded on considerations of physical and ontological simplicity. If we follow Earman's view, the violation of the principles in Lorentz's theory reflects in that the ether-and the corresponding privileged frame of reference the theory postulates - is empirically superfluous (I will return to this feature in the next section), suspicious of representing a 'nonintrinsic' reality, and implies asymmetries that do not correspond to the phenomena, as Einstein would put it.

\footnotetext{
${ }^{35}$ This is actually the case in Lorentz's theory. The Newtonian space-time structure postulated does not constrain the dynamical laws to express the same symmetries.

${ }^{36}$ Both Brown and Pooley, on one side, and Janssen and Balashov, on the other, are looking for constructive explanations in the kinematics vs. dynamical interpretation of special relativity. That is, even if we assume that theories of principle do explain, their debate presupposes that a full and physical explanation is given in constructive terms. If explanations of principle are possible, they only explain from a formal point of view, we could say, and in this sense an explanation of principle is ontologically subordinated to a constructive one. For an alternative in which the explanatory nature of special relativity is not absolute, but context-dependent, see (Dieks 2009).

${ }^{37}$ Brown $(2005$, p. 24$)$ argues that the substantivalist must assume that physical objects have 'space-time feelers' that allow them to react to space-time's 'ruts and grooves'.

${ }^{38}$ In a nutshell, Brown's dynamical interpretation of special relativity (see Brown 2005) consists in that if we take the Lorentz-invariance of dynamical laws as a given that need no further explanation-after all the laws of science explain the phenomena, but we usually do not demand for explanations of why the laws hold, or for why they have the form they havethen it follows that the kinematic, space-time structure will be Lorentz-invariant as well. This view, of course, is rather sympathetic to a relationist ontology concerning space-time. For an interesting assessment of the debate between BrownPooley and Janssen-Balashov, see (Frisch 2011). There the author argues that even though there is a real disagreement concerning the direction of the arrow of explanation between kinematics and dynamics in special relativity, there are more points of agreement than what it seems at first sight.
} 
Earman's argument to defend the first principle, namely, that the dynamical symmetries of a theory must also be space-time symmetries, is the following:

The motivation for (SP1) derives from combining a particular conception of the main function of laws of motion with an argument that makes use of Occam's razor. Laws of motion, at least in so far as they relate to particles, serve to pick out a class of allowable or dynamically possible trajectories. If (SP1) fails, the same set of trajectories can be picked out by the laws working in the setting of a weaker space-time structure. The theory that fails (SP1) is thus using more space-time than is needed to support the laws, and slicing away this superfluous structure serves to restore (SP1). (Earman 1989, pp. 46-7).

If we apply this argument to the case of Lorentz's ether theory, we have that the ether it postulates - and the associated privileged frame of reference - counts as the superfluous structure that is not required in order to support the laws (and as I will show below, it is also unnecessary for the derivation of empirical consequences) ${ }^{39}$. In this sense, special relativity is physically simpler than Lorentz's theory, for it does not postulate any superfluous space-time structure.

To justify his second principle - that the space-time symmetries must also be dynamical symmetriesEarman offers two arguments. The first one relies on general covariance and what he dubs 'nonintrinsic reality':

Let us say that the laws of $T$ are generally covariant just in case whenever $\mathbb{M}=\left\langle M, A_{1}, A_{2}, \ldots, P_{1}, P_{2}, \ldots\right\rangle \in$ $\mathfrak{M}_{T}$ [where $/ /$ stands for a model of the theory defined by the manifold $M$, the absolute objects $A_{i}$ geometric-object fields characterizing the fixed space-time structure-, the dynamic objects $P_{j}$ - geometricobject fields characterizing the physical contents of space-time-; and $\mathfrak{M}_{T}$ stands for the set of models of the theory], then also $\mathscr{M}^{\Phi} \in \mathfrak{M}_{T}$, where $\mathscr{M}^{\Phi} \equiv\left\langle M, \Phi * A_{1}, \Phi * A_{2}, \ldots, \Phi * P_{1}, \Phi * P_{2}, \ldots\right\rangle$ for any manifold diffeomorphism $\Phi$. Then if $\Phi$ is a space-time symmetry, i.e., $\Phi * A_{i}=A_{i}$ for all $i$, it follows from general covariance that $\Phi$ is a dynamical symmetry. Laws typically called generally covariant, i.e., partial differential equations written in terms of geometrical-object fields so as to have a form that is the same in every coordinate system, are ones that are generally covariant according to the above definition. The argument is completed by adding the premise that laws of motion, whatever their specific content and whether Newtonian, Einsteinian or otherwise, are about intrinsic reality that is independent of coordinate systems, observers, or point of view. The argument has noncircular force only to the extent that the latter premise can be supported by an independent characterization of intrinsic reality and a demonstration that a violation of general covariance involves a nonintrinsic reality'. (Ibid, p. 47).

To be applied to Lorentz's theory the argument must be somewhat adapted. In this case it is not general covariance what is at stake, but Lorentz-invariance. The Galilean transformations represent the symmetries of space-time in Lorentz's theory, but the dynamical laws are not invariant under these transformations. The upshot is that the Lorentz-invariant dynamical laws are local, in the sense that they determine the 'deceptive' measurements obtained in frames of reference that move across the ether. The dynamical laws determine the 'real' physics only in the case of the ether-rest frame. That is, using Earman's term, in the case of all the frames that are in motion with respect to the ether the dynamical laws refer to nonintrinsic reality.

Earman's second argument for the symmetry principle at issue

\footnotetext{
${ }^{39}$ More precisely, the ether and the related privileged frame of reference are the 'physical incarnations' of the Newtonian space-time structure of the theory — which, following Earman, is superfluous.
} 
derives from trying to imagine how (SP2) could fail. Presumably the theory would have to contain names, regarded as rigid designators, of regions of space-time, and the laws of the theory would say that the lawlike behavior that takes place in region $R_{1}$ is different from the lawlike behavior that takes place in $R_{2}$, even though $R_{2}=\Phi\left(R_{1}\right)$ for some space-time symmetry $\Phi$. But such a difference in lawlike behavior is reason to suppose that $R_{1}$ and $R_{2}$ differ in some structural property that grounds the difference in behavior. The characterization of this structural property in terms of the addition of new elements to the list of $A_{i}$ s means that $\Phi$ is no longer a space-time symmetry and (SP2) is restored. Putting the same point slightly differently, it is hard to see how to reconcile a violation of (SP2) with the widely accepted idea that laws of nature must be universal in the sense that the same laws hold good throughout space-time'. (Ibidem).

In the case of Lorentz's theory, this argument must also be somewhat reconsidered. In this theory the same laws of nature do hold throughout space-time - as we just saw, on the price of referring to "nonintrinsic reality'. However, the violation of SP2 implies that there is a different lawlike behavior in different regions - or more precisely, in different frames of reference - of space-time. The asymmetry that Einstein mentions in the 1905 relativity paper - the asymmetrical explanation of the electrodynamic interaction between a magnet and a conductor depending on whether we consider the former or the latter as at rest in the privileged frame - is a paradigmatic example.

I said that Earman's defense of the symmetry principle is grounded on considerations of physical and ontological simplicity in the sense that space-time theories that respect them are more economic: they do not contain superfluous structure, do not refer to nonintrinsic reality, and do not postulate ungrounded differences in lawlike behavior. In the case of Lorentz's theory, their violation entails that the ether-in connection with the privileged ether-rest frame and the Newtonian structure of space-time- - becomes suspicious of representing nothing physical. The fact that special relativity does not postulate entities or structures that are dubious in this sense makes it a physically and ontologically simpler theory than Lorentz's. That is, the comparative simplicity of Einstein's theory is not a merely pragmatic virtue, but it reflects more solid ontological foundations ${ }^{40}$.

To describe special relativity's physical simplicity in terms of Earman's symmetry principles has the advantage of allowing us to make sense of the historical role that such feature played in the acceptance of Einstein's theory. The asymmetries that the ether and a privileged frame of reference entailed was one of the motives determining Einstein's formulation of special relativity, and the physical simplicity of the theory was surely one of the reasons why it was accepted (over Lorentz's). In the previous section I argued that mathematical simplicity does not work as a criterion to make a choice, but it is very reasonable to think that the physical simplicity associated to the formulation of special relativity in terms of Minkowski space-time did not escape the eye of the scientists of the time. So when Laue, for example, refers to the simplicity

\footnotetext{
${ }^{40}$ Note that the conceptual justification to pick the observance of Earman's principle as a feature that shows special relativity's superiority over Lorentz's theory is not grounded on simplicity in and by itself, or as a merely aesthetic virtue. As I mentioned above, it is philosophically problematic to assert that simplicity is always desirable property in scientific theories. In this case, the simplicity manifested in the observance of Earman's symmetry principles reflects firmer ontological foundations.
} 
acquired by the four-dimensional geometric formulation, it is rather likely that he also had in mind the physical simplicity involved ${ }^{41}$.

These remarks thus show that the best way to reassess Janssen's argument concerning Lorentzinvariance is the following: the fact that special relativity respects the symmetry principles - whereas Lorentz's theory violates them - is a manifestation of a higher degree of physical simplicity. From the point of view of physical and ontological simplicity, Einstein's special relativity is clearly superior to Lorentz's ether theory, and this feature thus offers an objective reason in order to prefer the former over the latter.

\title{
3.4 The superfluous ether
}

The problematic status of the ether is another feature that has been typically considered as a reason to dismiss Lorentz's theory and to embrace special relativity. Usually, the ether is held to be problematic chiefly because it is undetectable. Since there is no possible observation that directly indicates the reality of the ether, we should simply apply Occam's razor and pick the most economic theory. Adolf Grünbaum, for example, has argued along this line:

\begin{abstract}
Since the observational consequences of the aether-theoretic interpretation of the Lorentz transformations are the same as those of their rival relativistic interpretation, the aether-theoretic interpretation can have no observational consequences which that are different from those of the rival special theory of relativity. Hence there can be no observational consequences which would support the doubly amended theory ${ }^{42}$ as against the new rival special theory of relativity, a theory that refuses to postulate the existence of some one preferred inertial aether frame when there is no kind of physical foundation for doing so. (Grünbaum 1973, p. 724; the last emphasis is mine).
\end{abstract}

Two objections can be leveled against this view. First, it is awkward to state that there was no physical foundation in order to postulate a preferred inertial ether frame. Maxwell's equations were originally understood as being valid in the ether-rest frame, and of course, this is the way in which Lorentz understood them. In his theory the ether-rest frame became physically privileged in order to account for the negative result of ether-drift experiments within the framework of a Newtonian space(-time) and classic electrodynamics. That is, the view that there was no physical foundation for the postulation of a preferred ether-rest frame is incompatible with historical facts. In the long run, it turned out that such postulation was unnecessary (or plainly wrong), but that is a different story.

Second, and more importantly, the automatic rejection of unobservable entities as a normative principle is debatable. Even if an entity postulated by a certain theory is directly undetectable, there still might be other observable effects which are connected to that entity, and in this sense they could be interpreted as

\footnotetext{
${ }^{41}$ Actually, Schaffner (1974, p. 74), in the passage by Laue he quotes, interprets the simplicity invoked in a physical way. Janssen (2002b, pp. 502-7) quotes Poincaré, Lorentz, Einstein and Minkowski in order to show that they explicitly discussed the foundations of Lorentz-invariance in connection to the Einstein vs. Lorentz case, and argues that such discussions can be described in terms of a COI argument. I think that we can make sense of all those references by means of physical simplicity, with the advantage of not getting involved in the epistemological troubles discussed above.

${ }^{42}$ What Grünbaum calls the doubly amended theory is Lorentz's core theory plus the length-contraction and the clockretardation hypotheses. As Janssen points out, the doubly amended theory is a sort of toy model that does not really grasp the historical subtleties in the development of Lorentz's theory. However, in this passage we can harmlessly replace 'the doubly amended theory' by 'Lorentz's definitive theory'.
} 
observable traces of its reality ${ }^{43}$. This is exactly what happens in Lorentz's theory. It is true that the ether is undetectable in this theory. However, the generalized contraction hypothesis is grounded in the interaction between physical systems and the ether. Therefore, all observable consequences in which this hypothesis is logically relevant for their derivation—such as the negative results of ether-drift experiments and the velocity-dependence of mass - can be understood as traces of the reality of the ether.

These remarks are enough in order to show that the standard arguments regarding the problematic status of the ether in Lorentz's theory are not compelling. However, there is a different perspective from which the ether can be questioned anyway. The real problem with it relies on the fact that, after Poincaré showed that the Lorentz coordinate transformations are symmetric, the ether became not only directly unobservable, but also empirically superfluous - a hypothesis is superfluous if it is logically irrelevant for the derivation of the empirical consequences of the theory it belongs to ${ }^{44}$.

In his 1906 On the Dynamics of the Electron, Poincaré demonstrated that if Lorentz's theory is to respect the principle of relativity, the Lorentz coordinate transformations must form a group. In turn, if the transformations form a group, in the inverse coordinate transformations the parameter $-v$ is replaced by $+v$, and the primed and unprimed quantities shift roles ${ }^{45}$. If this is so, the velocity parameter in the coordinate transformations refers to the relative velocities between the frames involved, not to the velocity with respect to the ether - this is clear if we apply the transformations in a case in which both frames are in motion with respect to the ether. Recall that in the definitive formulation of the theory its empirical consequences are derived from the coordinate transformations interlocked with the generalized contraction hypothesis. Since the velocity parameter in the symmetric Lorentz transformations is simply the relative velocity between the frames, reference to the ether is not logically required in order to derive the empirical consequences of the theory-its function becomes only theoretical. The only role that the ether plays is to provide the physical plausibility argument for the generalized contraction hypothesis. For example, in Lorentz's theory rods in motion contract because of their interaction with the ether, however, reference to the ether is not necessary to

\footnotetext{
${ }^{43}$ As Janssen puts it, 'as part of his analysis of the doubly-amended theory Grünbaum offers a more accurate diagnosis of the trouble with Lorentz's theory. What makes the theory unsatisfactory are not the elements that are added, but some of the original elements, notably the ether and Newtonian space-time, that are rendered more and more invisible with every amendment. This suggests that Ockham's razor is all that is needed to settle the case of Einstein versus Lorentz. The problem with this type of argument is that it derives its force from a blanket rejection of unobservables in scientific theories, whereas it is widely accepted that such elements should not be banned automatically. Rather than condemning unobservables in general, I think it is wiser to demand arguments to put forward on a case-by case basis to show why a particular unobservable is otiose' (Janssen 2002, p. 438). As I will show now, there is an argument that this particular unobservable is otiose.

${ }^{44}$ I take this concept of superfluity from (Norton 2008). This author argues that, given a pair of predictively equivalent theories, if one of them contains superfluous structure, this strongly suggests that the theories are, after all, one and the same. By excising the superfluous structure from the less economic theory we would obtain the empirically equivalent 'rival'. I think this not so in the case of Einstein vs. Lorentz. If we apply Occam's razor and excise the ether from Lorentz's theory, what we obtain is not special relativity. It is still possible to retain the Newtonian space-time plus conspiring dynamical effects by defining by fiat a privileged reference frame. For example, the Lorentzian could baldly say that the privileged frame is the one in which the real time is measured, period - even if it is impossible to physically determine which frame is that. That would be a theory with questionable foundations of course, but the point is simply that the main difference between special relativity and Lorentz's theory is based on the incompatible space-times they postulate. This difference remains even if we get rid of the ether.

${ }^{45}$ Recall that this feature is essentially required if Lorentz's theory is to be considered as empirically equivalent to special relativity. Curiously, Lorentz became aware of the symmetry of the transformations only after the work of Einstein. Lorentz knew Poincare's work of 1906, but he did not mention the Frenchman when he explicitly stated the symmetry of the transformations. See (Janssen 1995, section 3.5.4)
} 
predict that rods in motion get contracted (recall the example of rods $A$ and $B$ in section 2). In this scenario, to interpret the negative result of ether-drift experiments and the velocity-dependence of mass as traces of the reality of the ether becomes a dubious stance.

This unease in Lorentz's theory can thus be used to argue that special relativity is a better theory. By becoming empirically superfluous, the ether acquires a rather metaphysical flavor. As John Norton argues (2008, p. 35), superfluous structures or entities are highly suspicious of representing nothing physical. Within Einstein's theory, on the other hand, there is no such problem. The problematic status of the ether can thus also be described in terms of physical simplicity: the ontology of special relativity is more economic than the ontology of Lorentz's theory. Just as the violation of the symmetry principles, the superfluous character of the ether is a flaw in Lorentz's theory not (only) for aesthetic or pragmatic reasons. The comparative ontological simplicity of special relativity is connected to firmer ontological foundations.

\section{Empirical equivalence and theory choice: non-empirical features vs. empirical evidence}

This survey of the case of Lorentz vs. Einstein from the point of view of non-empirical features has delivered the following results. The accusation of ad-hocness, in spite of the main role it has played in the philosophical discussions about Lorentz's theory, has proven ungrounded. In the case of simplicity, the result is more subtle. If we consider simplicity from a mathematical point of view, we have that the fourdimensional language in which Minkowski presented Einstein's theory can also be used, mutatis mutandis, to formulate Lorentz's theory. Therefore, all the mathematical elegance and simplicity that the fourdimensional formulation of special relativity made explicit can also be assigned to Lorentz's ether theory. Physical simplicity, on the other hand, is a non-empirical feature that can certainly work as a criterion according to which special relativity proves superior. Its compliance with Earman's symmetry principles and the absence of the superfluous entity (the ether) make special relativity a simpler theory from an ontological point of view; and this comparative simplicity reflects that Einstein's is a theory with more solid foundations than its rival. We have thus found some non-empirical features that, at first sight, could justify a choice in the case of empirical equivalence we are considering.

However, non-empirical virtues cannot provide a fully objective and uniquely determined choice in cases of empirical equivalence. Theoretical virtues, such as simplicity and explanatory power, are usually context-dependent. That is, the specific degree of explanatory power or simplicity that a certain theory possesses depends on the context in which we are evaluating it. In the case of simplicity, we have that there are multiple senses in which a theory can be said to be simple, and these different forms of simplicity might be incompatible with each other (see Bunge 1961). Concerning explanatory power, as Van Fraassen (1980) argues, we have that explanations are answers to why-questions, so that the explanatory power of a certain theory depends on the specific why-question that is being addressed. Therefore, even if it is clear that a theory is simpler than and/or explanatorily superior to its predictively equivalent rival, there might be other senses in which the rival prevails. 
Moreover, there is no reason to presuppose that if a theory is superior to its predictively equivalent rival with respect to a certain non-empirical feature, that theory will be also superior with respect to all other theoretical aspects. Besides, there is no clear and universally accepted hierarchy between non-empirical features: is simplicity more important than explanatory power or vice versa? The value that is assigned to theoretical virtues typically depends on underlying epistemological credos, and, of course, different epistemological stances might result in different rankings of non-empirical features.

The upshot is that even though non-empirical features can provide good and justified reasons to choose one of the theories, it still might be the case that there are other contexts and/or other features according to which the opposite choice is also rational. In this sense, non-empirical features provide only a partial solution in cases of empirical equivalence, for they are not capable of providing a uniquely determined and fully objective choice ${ }^{46}$.

In the particular case of Lorentz vs. Einstein, we can imagine a supporter of Lorentz's theory that, when confronted with arguments concerning the explanatory superiority or the physical simplicity of special relativity, simply replies 'I accept that Einstein's theory scores better in those issues, however, Lorentz's theory — by means of the dynamic explanations in a Newtonian space-time it promises for effects like lengthcontraction, clock-retardation and the velocity-dependence of mass - offers a simple, classical and intuitive visualization that special relativity cannot provide'. As we saw above, Scott Walter has shown that the difficult intuitive spatio-temporal visualization involved in Minkowski space-time was noticed and considered by physicists like Laue and Wiechert. It is true that they traded the visualization issue for the elegance and simplicity of the Minkowskian presentation of Einstein's theory, but our Lorentzian physicist might not be willing to make that deal — and if it is to be argued that the only rational decision is to prefer special relativity, a previous demonstration that elegance and simplicity are more important features than intuitive visualization is required.

Lorentz's and Einstein's evaluation of their respective theories in the light of the constructive/principle distinction also shows that non-empirical features cannot yield a unique decision. In his 1919 article, Einstein explicitly evaluates his theory as a theory of principle:

The advantages of the constructive theories are completeness, adaptability and clearness, those of the principle theory are logical perfection and security of the foundations. The theory of relativity belongs to the latter class. In order to grasp its nature, one needs first of all to become acquainted with the principles on which it is based. (Einstein 1919, p. 228).

In the same article, Einstein states that constructive theories are explanatorily superior: 'when we say that we have succeeded in understanding a group of natural processes, we invariably mean that a constructive theory has been found which covers the process in question' (Ibidem). Lorentz's theory, on the other hand, is

\footnotetext{
${ }^{46}$ Non-empirical features ensure that the problem of empirical equivalence is not a threat for the rationality of theory choice. However, the fact that certain non-empirical features support a rational preference for one of the theories in a predictively equivalent pair does not mean that there are no other non-empirical features that can support a rational preference favoring the other theory. I claim that theoretical virtues offer only a partial solution of the problem of empirical equivalence and underdetermination in the sense that they block the threat on the rationality of theory choice, but they are not enough to provide a fully objective and uniquely determined choice.
} 
clearly a constructive one. Therefore, the problematic physical foundations on which it is built-recall the arguments regarding the comparative physical simplicity of special relativity—could be traded for the constructive explanation that it offers, explanations which are absent in Einstein's theory of principle ${ }^{47}$. Actually, something like this seems to have been what Lorentz had in mind when he evaluated the theories:

Einstein simply postulates what we have deduced, with some difficulty and not altogether satisfactorily, from the fundamental equations of the electromagnetic field. By doing so, he may certainly take credit for making us see in the negative result of experiments like those of Michelson, Rayleigh and Brace, not a fortuitous compensation of opposing effects but the manifestation of a general and fundamental principle.

Yet, I think, something may also be claimed in favour of the form in which I have presented the theory. I cannot but regard the ether, which can be the seat of an electromagnetic field with its energy and its vibrations, as endowed with a certain degree of substantiality, however different it may be from all ordinary matter. In this line of thought it seems natural not to assume at starting that it can never make any difference whether a body moves through the ether or not, and to measure distances and lengths of time by means of rods and clocks having a fixed position relatively to the ether. (Lorentz 1916, pp. 229-30, my emphasis).

More generally, the fact that Lorentz's theory offers an account of the phenomena that allows us to remain within the framework of classic electrodynamics and Newtonian space-time might be enough for the Lorentzians to simply dodge the fact that special relativity is a better theory with respect to other nonempirical features. All that is needed to defend such a position is to underscore that the ether and the preferred frame of reference in Lorentz's theory allow to have a physical seat for the electromagnetic fields — as Lorentz states in the quoted passage — and an intuitive, 'classic' visualization of length-contraction and clock-retardation. To highly value these epistemic features is a position that can be, of course, defended ${ }^{48}$.

If non-empirical features is all what we have for deciding the case of Einstein vs. Lorentz, then the decision cannot be determined in a fully objective and unique way. We would be in a sort of Kuhnian

\footnotetext{
${ }^{47}$ As mentioned above, the question of the correct interpretation of special relativity — constructive or principle, kinematic or dynamic - is an ongoing debate. This is already enough to state that the explanatory features of Einstein's theory are not sufficient to decide a choice in the case of empirical equivalence we are considering. However, just in order to show that, in principle, the superiority of special relativity in a given non-empirical feature can be compensated in Lorentz's theory, we can follow Einstein's opinion that special relativity is a theory of principle in which the relativistic effects are kinematically explained, without invoking casual processes and atomic microstructure. In a letter to Arnold Sommerfeld, dated January 1908, Einstein expresses his concern about special relativity's lack of constructive-dynamical explanation: 'So, first to the question of whether I consider the relativistic treatment of, e.g, the mechanics of electrons as definitive. No, certainly not. It seems to me too that a physical theory can be satisfactory only when it builds up its structures from elementary foundations. The theory of relativity is not more conclusively and absolutely satisfactory than, for example, classical electrodynamics was before Boltzmann had interpreted entropy as probability. If the Michelson-Morley experiment had not put us in the worst predicament, no one would have perceived the relativity theory as a (half) salvation. Besides, I believe that we are still far from having satisfactory elementary foundations for electrical and mechanical processes. I have come to this pessimistic view mainly as a result of endless, vain efforts to interpret the second universal constant in Planck's radiation law in an intuitive way. I even seriously doubt that it will be possible to maintain the general validity of Maxwell's equations for empty space' (quoted in Brown 2005, pp. 72-3).

${ }_{48}$ During lectures he gave in Leiden in 1910-12, published in 1922, Lorentz describes the advantages in his theory- the ether as the carrier of electromagnetic fields and classic chrono-geometry - in the following way: "whether there is an aether or not, electromagnetic fields certainly exist, and so also does the energy of oscillations. If we do not like the name of "aether", we must use another word as to peg to hang all these things upon. It is not certain whether 'space' can be so extended as to take care not only of the geometrical properties but also of the electric ones.

One cannot deny to the bearer of these properties a certain substantiality, and if so, then one may, in all modesty, call true time measured by clocks which are fixed in this medium, and consider simultaneity as a primary concept' (quoted in Brown 2005, p. 66).
} 
conundrum, in the sense that the decision could be made only by presupposing certain (non-empirical) standards of theory appraisal, standards which are not shared by the whole scientific community. And since the theories are predictively equivalent, the merits of the competing standards of theory appraisal cannot be evaluated in terms of empirical success. If this Kuhnian riddle were the case, the current situation in the scientific community concerning regarding the acceptance or rejection of special relativity and Lorentz's theory should be analogous to the case of Bohm's theory and standard quantum mechanics. Even though there is a clear orthodoxy favoring the latter theory, there is a small dissenting group of supporters of the former - but such a group is not a crew of stubborn, or simply irrational, scientists. However, Lorentz's theory is not supported by any group of respectable physicists, its importance is nowadays only historical and philosophical $^{49}$.

\section{Einstein vs. Lorentz in the light of non-consequential empirical evidence}

In this section I will argue that, in spite of the limitations of non-empirical features as possible solutions for the problem of empirical equivalence, the case of Einstein vs. Lorentz can be decided in a fully objective and uniquely determined way, if we have recourse to non-consequential empirical evidence. Given the predictive equivalence of the theories, the deciding evidence cannot be given by the observational consequences of the theories. I will briefly refer to arguments by Larry Laudan and Jarret Leplin (1991) and Richard Boyd (1973), in order to show that, if we accept certain noncontroversial principles of confirmation, it follows that non-consequential empirical evidence can provide an objective and uniquely determined decision in cases of empirical equivalence. I will argue that such non-consequential evidence can be invoked in the particular case of Einstein vs. Lorentz, and also that the historical course of events that led to the abandonment of the latter and the acceptance of the former can be understood from this point of view.

\subsection{Lorentz's theory and quantum physics}

The first principle of non-consequential evidence that can be used in order to decide the case of empirical equivalence we are considering relies on an argument proposed by Richard Boyd (1973). This author refers to the Reichenbach-Poincaré argument for the conventionality of geometry. This argument presents us two empirically equivalent theories $T$ and $T^{\prime} . T$ describes the physical world by means of a set of

\footnotetext{
${ }^{49}$ It must be underscored that the fact that non-empirical features are not enough to provide a definitive and universally accepted decision in cases of empirical equivalence does not imply that theoretical virtues as simplicity or explanatory power are merely pragmatic issues that are not relevant in contexts of theory choice, or that they are not at all connected to empirical evidence. For example, James McAllister (1989) argues that 'indicators of beauty' (non-empirical virtues) are grounded on a meta-induction on theoretical features of empirically successful theories. In spite of this connection, McAllister states that 'the hope that indicators of beauty will defeat the threat of underdetermination is incidentally revealed illusory: any decision on aesthetic grounds between empirically equivalent theories will in general be perceived as valid only within the paradigm then current and cannot hence be considered definitive' (McAllister 1989, p. 44)-McAllister uses a temporal language because he describes scientific revolutions, in the Kuhnian sense, in terms of radical changes in the canons that define indicators of beauty; but we can adapt the quote to our context and replace 'the paradigm then current' for 'a presupposed paradigm'.
} 
forces $F$ and a geometry $G$, and $T^{\prime}$ postulates a set of forces $F^{\prime}$ and a geometry $G^{\prime}$-where $F^{\prime}$ includes all the forces in $F$ plus a 'universal' force $f^{\prime 50}$. Boyd argues that

even though " $F \& G$ " and " $F$ ' \& $G^{\prime \prime}$ " have the same observational consequences (in the light of currently accepted theories), they are not equally supported or disconfirmed by any possible experimental evidence. Indeed, nothing could count as experimental evidence for " $F$ ' \& $G^{\prime}$ " in the light of current knowledge. This is so because the force $f^{\prime}$ required by $F^{\prime}$ is dramatically unlike those forces about which we know-for instance, it fails to arise as the resultant of fields generating in matter or in the motions of matter. Therefore, it is, in the light of current knowledge, highly implausible that such a force $f^{\prime}$ ' exists.

Furthermore, this estimate of the implausibility of " $F$ ' \& $G$ "' reflects experimental evidence against " $F$ ' \& $G$ "', even though this theory has no falsified observational consequences (Boyd 1973, pp. 7-8).

From Boyd's account of this example we can extract a general principle of empirical evidence. Given two empirically equivalent theories, the corpus of accepted, well-confirmed background knowledge might be (or become) at odds with the theoretical core of one of the two theories, but (remain) coherent with the other one. The friction between accepted background knowledge, or, more precisely, between the experimental evidence that supports background knowledge and the theoretical-core of one of the predictively equivalent theories can count as empirical, non-consequential evidence to reject such theory ${ }^{51}$.

It is important to remark that this standard of non-consequential empirical evidence relies on an epistemological stance: that consistency between the theories we accept is a basic principle of science. Suppose that theories $T$ and $T$ ' are predictively equivalent, that $T$ is consistent with another well-confirmed theory $P$, and that $T$ ' is at odds with it. The evidential support for $P$ counts as empirical evidence against $T$, granted that we agree that consistency between the theories we accept is a basic principle of science. If we want that our theories are mutually consistent, then the standard of non-consequential evidence just introduced can be taken as a principle in the dynamics of empirical confirmation.

In order to clearly recognize that this consistency principle is a basic tenet of science it is enough to imagine a case in which two well-confirmed theories are mutually inconsistent. Suppose that in a certain domain of physics theory $T$ is introduced and that all of its predictions are observed, that in a different domain theory $P$ is proposed and all its predictions are confirmed, and that $P$ and $T$ are blatantly incompatible. This situation, of course, would be taken as a serious problem for science, and it would be expected that endeavors in order to show that one of the theories must be given up, or endeavors to formulate

\footnotetext{
${ }^{50}$ A universal force, roughly speaking, is a force that acts equally on all physical objects and that it cannot be shielded against. A differential force, on the contrary, can be shielded against and does not act equally on all physical objects. See (Reichenbach 1958, §6). The argument asserts the conventionality of physical geometry insofar as the empirical equivalence between $T$ and $T^{\prime}$ is supposed to imply that the choice between $G$ and $G^{\prime}$ is just a matter of convention.

${ }^{51}$ Boyd's own position is that, in a case of empirical equivalence between $T$ and $T^{\prime}$, the compliance of $T$ with the form of causal explanations present in empirically successful theories in background knowledge counts as an indicator for the truth of $T$ that is lacking in $T^{\prime}$-for the explanations in $T^{\prime}$ do not have the mentioned form. The principle of confirmation I just defended weakens Boyd's position in the sense that it is detached from any realist commitments (he considers the problem of empirical equivalence and underdetermination as a threat to the realist, but I consider it as a matter of theory choice), and at the same time generalizes Boyd's proposal in the sense that possible friction with background knowledge is not given only by divergence from the canonical form of causal explanations. That is, I am using his argumentation to extract a principle of nonconsequential evidence that can be applied to the case of Einstein vs. Lorentz, but I do not intend to vindicate his views on realism or causality.
} 
a more fundamental (consistent) theory that encompasses both $P$ and $T^{52}$, would be undertaken by scientists. The conceptual, epistemic justification for the principle is also easy to provide. If we aspire to obtain knowledge of reality by means of scientific theories, it is clear that if the set of scientific theories we accept is inconsistent, we would hardly call such set 'knowledge'. To say that 'we know that $2+2=4$ and that $2+2=5^{\prime}$ is clearly nonsense $\mathrm{e}^{53}$.

We can apply the principle of non-consequential empirical evidence just explained to the case of special relativity vs. Lorentz's theory if we consider the relation of the latter with early quantum physics. In 1899 Max Planck introduced the concept of the quantum of energy in order to derive the correct law for blackbody radiation. Before Planck's work, the black-body radiation spectrum had been an intractable problem for classic electrodynamics and thermodynamics. Planck's quantum hypothesis was soon acknowledged to be deeply at odds with the foundations of electrodynamics. In a nutshell, the problem was that classic electrodynamics and thermodynamics predict that an accelerated electron must emit radiation of all wavelengths in a continuous range of energy, whereas the quantum hypothesis postulated emission in determinate, specific wavelengths in a discrete spectrum. Since Lorentz's ether theory-and the model of the electron it included —-was essentially built upon the very core of classic electrodynamics, namely, Maxwell's equations, the groundbreaking new physics of the quantum led physicists to gradually abandon it. The problem got even deeper with Bohr's first contributions on the structure of the atom, for the quantum hypothesis and the abandonment of classical electrodynamics were central features in Bohr's work.

Actually, Hendrik Lorentz himself played a central role in the recognition of the essential conflict between quantum physics and the core of classic electrodynamics (see Kox 2013 and McCormmach 1970, pp. 486-7). Lorentz, between 1900 and 1903, devoted his work to find the expression for density radiation of black-body as a function of its temperature and the wavelength of the radiation. The formula he obtained on the basis of electrodynamics - using his model of the electron — applied to thermodynamics was equivalent to the Rayleigh-Jeans law, and, of course, it worked only in the long-wavelength part of the spectrum of emission $^{54}$. Although he first took this result as very promising, he soon realized that his model of the electron - a cornerstone of his ether theory - and electrodynamics in general were at deep odds with the results of black-body experiments and the quantum hypothesis required in order to explain such results:

In 1908 Lorentz came out in support of Planck's theory; it was then that he emphasized the profound antithesis between the quantum hypothesis and the electron theory. At a mathematical congress in Rome that year Lorentz spoke on Planck's and James Jeans' theories of blackbody radiation. His object was to prove that the union of the electron theory with Hamilton's equations of motion and J. W. Gibbs' statistics leads

\footnotetext{
${ }^{52}$ Actually, this is more or less the situation concerning the conflict between general relativity and relativistic quantum theory.

${ }^{53}$ It must be mentioned, though, that one of the motivations for the development of paraconsistent logic was to deal with (possible) inconsistencies between or within physical theories.

${ }^{54}$ 'Lorentz pointed out that his black-body formula agrees with the long wavelength limit of the quantum formula that Planck had derived in 1900, a coincidence which struck him as highly remarkable considering the widely different assumptions in the two cases. It was characteristic of Lorentz to spell out what was incomplete in his work and what was still unknown; he stressed that his theory is valid only for long wavelengths and that Planck's applies to the whole spectrum. So it was Lorentz, an originator of the electron theory, who first intimated the possible limits of the theory. Starting from the electron theory and from a mechanism appropriate to the theory, he arrived at the limiting case of the radiation law; and he did not see how to extend his theory to Planck's general case.' (McCormmach 1970, pp. 486-7).
} 
inescapably to Jeans' radiation law, which, like his own of 1903, agrees with experience only in the case of long wavelengths. He said that the alternative, Planck's theory, demands far-reaching changes in electron theory. He pointed out that this is easily seen, since an accelerated electron should emit rays of all wavelengths, a result incompatible with the hypothesis of energy elements whose magnitude depends on wavelength. At the time of his lecture he had not yet decided between the two theories. Wien, however, called his attention to experiments showing that for short wavelengths a body emits much less light in proportion to its absorbing power than that predicted by Jeans' theory. This proves, Lorentz said in a note appended to the published version of his talk, that any theory that bases itself on the electron theory and the equipartition theorem has to be profoundly revised. Later in that year he elaborated that note: he had 'long hoped', he confessed, 'that it would be possible to escape the universal applicability of that theorem [equipartition] by combining electron theory and kinetic theory'. He added, 'this hope has not been fulfilled'. He was now ready to concede that the interaction of matter and ether takes place by means of vibrating charged particles to which Gibbs' statistics, for unknown reasons, are inapplicable.

Lorentz thus accepted the quantum theory as the only theory capable of explaining the complete spectrum of black-body radiation, while at the same time regarding it as very incompletely understood in its connection with the other branches of physics and in particular with electron theory. (Ibid, p. 487).

This conflict between electrodynamic theory and quantum physics, that Lorentz himself contributed to make explicit, became more and more important in the scientific discussion during the following years. It was eventually realized that Lorentz's electrodynamic model of the electron was essentially incompatible with the new physics of the quantum ${ }^{55}$. This recognition, in turn, worked as one of the main reasons why Lorentz's theory was abandoned, leaving the way open for the prevalence of special relativity:

The sense of the first Solvay Congress in 1911 was that the electron theory was incompatible with quanta and that it could not be made compatible without far reaching reform. The Congress and especially its published proceedings went far to redefine the fundamental problems for fundamental physical theory. Niels Bohr's doctoral dissertation in 1911 was a reformulation of Lorentz's theory of metals on more general principles. In his dissertation Bohr pointed to persuasive evidence of the ultimate incompetence of mechanics and electrodynamics on the molecular level. His 1913 quantum theory of atoms and molecules, which gave sharp focus to the quantum problems and intimated their enormous fruitfulness, was based on the explicit denial of the validity of ordinary mechanics and the classical electron theory in the atomic domain. Lorentz's theory continued to be worked on, but its concepts were increasingly recognized as unsuited for the basic reconstruction of physical theory demanded by the quantum hypothesis. (McCormmach 1970, p. 488).

On the other hand, special relativity, in spite of its predictive equivalence with Lorentz's theory, was not in conflict with the quantum hypothesis. Einstein's theory, as we saw above, is a theory of principle, and as such, it is silent with respect to issues concerning the ultimate structure of matter, fields or energy. The conflict of Lorentz's theory with quantum mechanics was grounded on the model of the electron included in the former. In special relativity there simply was no model of the electron at all. This feature became totally explicit in the eyes of the scientific community of the times through the work of Minkowski. This essential difference between the theories was not initially noticed by the scientific community. Actually, the expression Lorentz-Einstein theory was common before Minkowski's famous conference of 1908-

\footnotetext{
${ }^{55}$ Lorentz's ether theory is not a theory about thermal phenomena, of course. However, the main tenets of the electron model it includes can be applied to thermodynamics — as Lorentz did between 1900 and 1903. Thus, the observed spectrum of emission of black-body radiation does not directly falsify the ether theory, but Planck's law cannot be derived using Lorentz's model of the electron. The theoretical assumptions required for the derivation of Planck's correct law of black-body radiation are thus incompatible with the ether theory.
} 
published in 1909. There he showed that special relativity is a theory grounded on the kinematics of the fourdimensional continuum - Minkowski space-time - and this made superfluous any specific assumptions about dynamics. This difference between Einstein's and Lorentz's theory was crucial for the abandonment of the latter and the acceptance of the former.

Considering that quantum physics became more and more important during the first two decades of the $20^{\text {th }}$ century, it was rather natural that, given two predictively equivalent theories, the one which was not at odds with quantum theory was to be accepted. Indeed, by 1909 the expression Lorentz-Einstein theory began to disappear from physicists' vocabulary. Minkowski's work had shown that they were two theories of a different nature, and by 1911 the rise of quantum mechanics turned the balance decidedly in Einstein's favor. As Helge Kragh explains in his excellent book on the history of $20^{\text {th }}$ physics:

Why did the electromagnetic program run out of power? [...] More important was the competition from other theories that were either opposed to the electromagnetic view or threatened to make it superfluous. Although the theory of relativity was sometimes confused with Lorentz's electron theory or claimed to be compatible with the electromagnetic worldview, about 1912 it was evident that Einstein's theory was of a very different kind. It merely had nothing to say about the structure of electrons and with the increasing recognition of the relativistic point of view, this question - a few years earlier considered to be essential — greatly changed in status. To many physicists it became a pseudo-question. As the rise of relativity made life difficult for electromagnetic enthusiasts, so did the rise of quantum theory. Around 1908, Planck reached the conclusion that there was a fundamental conflict between quantum theory and the electron theory, and he was cautiously supported by Lorentz and other experts. It seemed that there was no way to derive the blackbody spectrum on a purely electromagnetic basis. As quantum theory became more and more important, electron theory became less and less important. The worst thing that can happen to a proclaimed revolution is that it is not needed. (Kragh 1999, p. 115).

Let us recall the structure of the principle of non-consequential empirical evidence introduced above. Consider two predictively equivalent theories $T$ and $T^{\prime}$, such that the theoretical core of $T^{\prime}$ is at odds with theory $P$, which is empirically well-confirmed, whereas $T$ and $P$ are compatible. Our principle indicates that, given the conflict, the empirical evidence supporting $P$ counts as evidence against $T^{\prime}$, but is neutral with respect to $T$. Therefore, in spite of their empirical equivalence, theory $T$ has more evidential support than $T^{\prime}$. After what has been said in the previous paragraphs, we can simply replace $T$ for 'special relativity', $T^{\prime}$ for 'Lorentz's theory', and $P$ for 'early quantum physics'. In other words, the case of Lorentz vs. Einstein can be decided in terms of non-consequential empirical evidence.

Notice that this view allows us to make sense of the case of predictive equivalence at issue both from the conceptual and the historical point of view. We have just seen that the decision made by the scientific community of the time was crucially determined by the conflict between Lorentz's theory and quantum physics. The unease between the latter and electrodynamics was historically relevant also in the formulation of special relativity. Einstein was fully aware of the deep crisis that the quantum hypothesis was about to provoke. With particular foresight, just after the publication of Planck's work he noticed that the quantum hypothesis was to lead to a deep reformulation of the basic framework of physics. Furthermore, a few months before his paper on special relativity appeared, Einstein published his work on the light-quantum 
hypothesis - hypothesis which relied on Planck's concept — so the fact that electrodynamics was going to dramatically crash against the new physics of the quantum was particularly clear in front of Einstein's eyes ${ }^{56}$. This awareness, in turn, was a crucial factor in the formulation of special relativity. The theory to be created had to elude the quantum conflict, and the way to achieve that was to avoid commitments to electrodynamic models, that is, by an approach of principle:

Reflections of this type [the quantum-issues] made it clear to me as long ago as shortly after 1900, i.e., shortly after Planck's trailblazing work, that neither mechanics nor thermodynamics could (except in limiting cases) claim exact validity. By and by I despaired of the possibility of discovering the true laws by means of constructive efforts based on known facts. The longer and the more despairingly I tried, the more I came to the conviction that only the discovery of a universal formal principle could lead us to assured results. The example I saw before me was thermodynamics. The general principle was there given in the theorem: the laws of nature are such that it is impossible to construct a perpetuum mobile (of the first and second kind). (Einstein 1959, pp. 51-3).

I mentioned in section 3.1 that, even though Schaffner recognized the crucial relevance of quantum physics in the case of Einstein vs. Lorentz, his conceptual approach was misguided. First, since he describes the matter in terms of the concept of $\mathrm{ad} \mathrm{hoc}_{3}$, the reason to prefer special relativity over the ether theory gets grounded on a non-empirical feature. If this is so, then the limitations of non-empirical features in cases of empirical equivalence I explained in section 4 hold also for Schaffner's proposal. Lorentzian scientists could still argue 'OK, our theory is ad $\operatorname{hoc}_{3}$, but we still have good reasons to support it'. However, historical facts show that the friction with quantum mechanics was a fatal blow for classic electrodynamics, including Lorentz's theory. The definitive impact of the quantum hypothesis on the outcome of the case of Lorentz vs. Einstein gets thus better described in terms of non-consequential empirical evidence. Since the ultimate tribunal of theory choice favored special relativity, then non-empirical virtues in Lorentz's theory could not be invoked any more in order to defend it and support it $^{57}$.

\footnotetext{
${ }^{56}$ Einstein narrated this episode with particular clarity in his Autobiographical Notes: 'Planck got his radiation-formula if he chose his energy elements $\varepsilon$ of the magnitude $\varepsilon=h v$. The decisive element in doing this lies in the fact that the result depends on taking for $\varepsilon$ a definite finite value, i.e., that one does not go to the limit $\varepsilon=0$. This form of reasoning does not make obvious the fact that it contradicts the mechanical and electrodynamical basis, upon which the derivation depends. Actually, however, the derivation presupposes implicitly that energy can be absorbed and emitted by the individual resonator only in "quanta" of magnitude $h v$, i.e., that the energy of a mechanical structure capable of oscillations as well as the energy of radiation can be transferred in such quanta - in contradictions to the laws of mechanics and electrodynamics. [...] All of this was quite clear to me shortly after the appearance of Planck's fundamental work; so that, without having a substitute for classical mechanics, I could nevertheless see to what kind of consequences this law of temperature-radiation leads, as well as for other related phenomena of the transformation of radiation-energy, as well as for the specific heat of (especially) solid bodies. All my attempts, however, to adapt the theoretical foundation of physics to this [new type of] knowledge failed completely. It was as if the ground had been pulled out from under one, with no firm foundation to be seen anywhere, upon which one could have built' (Einstein 1959).

${ }^{57}$ Unlike Schaffner, Janssen does not attribute the rise of quantum physics a crucial important in the resolution of the case we are considering: 'According to this electromagnetic program, the common origin underlying universal Lorentz invariance is that all matter is made of electromagnetic fields and is thus governed by Maxwell's equations. Since Maxwell's equations are Lorentz invariant, all systems in motion must contract. The program initially showed promise, but it did not pan out. Special relativity thus carried the day, but not thanks to this or any other COI. Variants of the COI laid out above, in conjunction with arguments from nascent quantum theory, removed Lorentz's theory from serious consideration, but did not decide between special relativity and the electromagnetic theory' (Janssen 2002b, p. 499). I disagree with the last sentence. As I just argued, the empirical confirmations of early quantum theory provided empirical evidence to reject Lorentz theory, whereas that evidence was neutral with respect to special relativity.
} 
Before we consider a second way in which non-consequential evidence can settle the case of Einstein vs. Lorentz, it is interesting to mention that the previous remarks show that, after all, explanatory issues are conceptually and historically relevant. However, the explanatory features of special relativity are significant to make a definitive choice if we consider them not only as non-empirical features. The (kinematic) explanations of principle of special relativity are superior to the (dynamic) constructive explanations of Lorentz's theory because the former are not in conflict with the quantum hypothesis and its empirical confirmations $^{58}$. That is, the explanatory superiority of Einstein's theory is grounded on the fact that the kind of explanations it offers do not lead to empirical problems ${ }^{59}$.

\subsection{Special and general relativity}

The second principle of non-consequential evidence that can be invoked in the case of empirical equivalence we are considering relies on an argument proposed by L. Laudan and J. Leplin (1991). These authors - in the same vein as Boyd - argue that if we hold on to two basic and very plausible principles of confirmation, then it follows that the class of observational statements that can confirm a theory does not reduce to the class of observational consequences that theory entails.

Suppose that a theory $T$ implies two logically independent theoretical hypotheses $H_{1}$ and $H_{2}$, and in turn $H_{1}$ entails the observational consequence $e_{1}$. Then, if $e_{1}$ is true it counts as empirical evidence for $H_{1}$, for $T$, and also for $H_{2}$, even though $e_{1}$ is not a logical consequence of $H_{2}$. This confirmation scheme relies on the two principles that Laudan and Leplin invoke. The first principle states that evidence for a hypothesis $h$ is also evidence for the statements that imply $h^{60}$ - this is why in our example $e_{1}$ confirms $T$. The second principle says that evidence for a hypothesis $h$ is also evidence for the statements that $h$ entails ${ }^{61}$ - this is the reason why $e_{1}$ confirms $\mathrm{H}_{2}{ }^{62}$.

We can now apply the confirmation scheme and the principles just explained cases of empirical equivalence. As Laudan and Leplin show, the standard of non-consequential evidence involved can be the source for a decision in such cases:

\footnotetext{
${ }^{58}$ If we want to avoid to get troubled by the dispute about the correct interpretation of special relativity, we can simply omit the adjectives 'kinematic' and 'of principle'.

${ }^{59}$ After the completion of this work a came into a paper by Nugayev (1985) that also refers to the friction between Lorentz's theory and early quantum physics. However, this author argues that it was not special relativity what defeated Lorentz's theory, but that it was Einstein's scientific research program — where he understands 'research program' in a strictly Lakatosian sense. Nugayev argues that not only special and general relativity can be described as the results of the heuristics of the same research program, but also that Einstein's work on the light-quantum can be included in it. It is already dubious that Einstein's road from special to general relativity can be described in terms of Lakatos' model, let alone the inclusion of the light-quantum hypothesis. Even worse, Nugayev does not offer any argument in order to support the view that the lightquantum hypothesis was a stage in the development of 'Einstein's research program'.

${ }^{60}$ Hempel (1945, p. 104), dubs this principle 'the converse consequence condition'.

${ }^{61}$ This is Hempel's 'special consequence condition' (Ibid., p. 103).

${ }^{62}$ By defending this scheme of confirmation I am not presupposing that evidence is just a matter of logical relations. That is, one could still come up with the question 'how good an evidence is $e_{1}$ for $H_{2}$ ?' The pattern of evidence just sketched only means that $e_{1}$ can in general be evidence for $\mathrm{H}_{2}$, but how good is that evidential support might depend on contextual features or on the specific meanings of the statements involved, for example. The important point here is that, in the case of empirical equivalence we are considering, the statement corresponding to $e_{1}$ does provide good evidence for the hypothesis corresponding to $\mathrm{H}_{2}$, as we will see.
} 
Theoretical hypotheses $H_{1}$ and $H_{2}$ are empirically equivalent but conceptually distinct. $H_{1}$, but not $H_{2}$, is derivable from a more general theory $T$, which also entails another hypothesis $H$. An empirical consequence $e$ of $H$ is obtained. $e$ supports $H$ and thereby $T$. Thus, $e$ provides evidential warrant for $H_{1}$, of which it is not a consequence, without affecting the credentials of $H_{2}$. (Laudan and Leplin 1991, p. 464).

The two underlying principles are quite sound as providing standards of confirmation. For example, in his classic paper on confirmation, Carl Hempel considers them as logical requirements that any plausible account of confirmation has to satisfy $(1945 \text {, p. } 102 \text { and ff. })^{63}$. On the other hand, it is not difficult to realize that these principles are actually applied in scientific practice. If we reject them, many paradigmatic cases of confirmation of theories by empirical evidence should be queried.

The way in which this second scheme of non-consequential evidence can be applied to the case of empirical equivalence at issue is by referring to the inter-theoretical connection between special relativity and general relativity. In 1916 Einstein finished his general theory of relativity. In this gravitational theory, the Minkowski space-time described by special relativity becomes a special case. General relativity tells us that Minkowski space-time depicts an infinite universe devoid of mass-energy, and that Minkowski geometry is the geometry of the tangent space-time attached to every point of a space-time defined by general relativity_ just as in Gaussian geometry the geometry of the tangent plane associated to every point of a curved surface approximates to the geometry of a Euclidean plane. That is, special relativity becomes a special case of the general theory, both in the mathematical and the physical sense.

Lorentz's theory — in spite of its predictive equivalence with respect to special relativity — does not fit within general relativity. As mentioned above, it claims that the physical world, in its spatiotemporal features, has the structure of Newtonian space-time. Therefore, even if we take Lorentz's theory as holding for a local region of a global space-time, it is in conflict with the meaning of general relativity. Einstein's gravitational theory states that the geometry of an infinitesimal region of a global space-time does not approximate to the geometry of a Newtonian space-time, but to the geometry of a Minkowski space-time. Despite the mathematical and empirical equivalence between Lorentz's theory and special relativity, the former cannot be understood as a special case of general relativity; the theories are incompatible.

As a result, special relativity possesses greater empirical support than Lorentz's ether theory. General relativity entails predictions that special relativity cannot entail on its own - it allows a satisfactory

\footnotetext{
${ }^{63}$ It is important to mention that, as Hempel immediately noticed, the unqualified acceptance of these two principles leads to a logical problem, as Samir Okasha puts it, 'The absurdity that results is this: every statement confirms any other one. For consider any statement $S$. Every statement confirms itself, so $S$ confirms $S$. By converse consequence, $S$ confirms $(S \& T)$, since $(S \& T) \rightarrow S$. By special consequence, $S$ confirms $T$, since $(S \& T) \rightarrow T$. This result holds for arbitrary $T$, and must therefore be regarded as a reduction ad absurdum of the simultaneous use of the special and converse consequence conditions (Okasha 1997, p. 253). Hempel's solution consisted in that the converse consequence condition should not be accepted as a universal rule of confirmation, but only in cases in which the relation between the stronger and the weaker hypotheses is such that the latter is 'essentially a substitution instance of the stronger one' (Hempel 1945, p. 104-5). For example, Galileo's law of free falling is, according to Hempel, a substitution instance of Newton's law of gravitation. I think that a better solution is the following. First, we can apply the converse consequence condition to Okasha's $S$ \& $T$ only if both statements are genuinely scientific hypotheses. Otherwise, theologists could conjunct the statement 'god exists' to Einstein's principle of equivalence, and thus the existence of god would be confirmed by the observation of gravitational time-dilation. Second, we can apply the converse consequence condition to $S \& T$ only if this conjunction allows to predict or explain phenomena that neither $S$ nor $T$ in isolation could explain or predict. Otherwise, cosmologists could conjunct string theory to genetics and claim that the discovery of a new gen confirms that space-time has eleven dimensions. The advantage of this solution is that, unlike in Hempel's, we do not need to a priori restrict the kind of logical relation between the stronger and the weaker hypotheses.
} 
description of the motion of the perihelion of Mercury, and it predicts that light gravitates and 'bends', for example. However, since the special theory is a special case of the general one, the empirical support for the latter flows to the former: the perihelion of Mercury and the light-bending effect provide empirical evidence for general relativity, therefore, those phenomena are also evidence for the view that the Minkowski geometry is the geometry of the tangent space-time associated to each point of a global space-time. On the other hand, since Lorentz's theory is incompatible with general relativity and thus cannot be included in it, the empirical support of Einstein's gravitational theory cannot flow to Lorentz's ether theory.

It is not difficult to notice that the inter-theoretical relation between special and general relativity (and Lorentz's theory) instantiate the standard of non-consequential empirical evidence proposed by Laudan and Leplin. Recall that if we have two empirically equivalent hypotheses $H_{1}$ and $H_{2}$, such that $H_{1}$ - but not $H_{2}-$ can be embedded in a more general theory $T$, and if $T$ is such that it entails the hypothesis $H$, which in turn implies the observational statement $e$; then the truth of $e$ counts as empirical evidence for $H$, for $T$ (converse consequence condition), and for $H_{1}$ (special consequence condition), but not for $H_{2}$. After what has been said about the relations between special relativity, general relativity and Lorentz's theory it is easy to see that $H_{1}$ can be replaced by 'special relativity', $H_{2}$ by 'Lorentz's theory', $T$ by 'general relativity', $H$ by 'light gravitates', and $e$ by 'the results of Eddington's expedition'. Therefore, the theoretical interconnections described imply that, despite their predictive equivalence, special relativity has more evidential support than Lorentz's theory ${ }^{64}$.

As mentioned above, the acceptance of Einstein's theory and the abandonment of Lorentz's by the scientific community of the time occurred around 1911. That is, by 1919-the year when the bombastic acceptance of general relativity produced by Eddington's observation of the 'light-bending' effect— the case

\footnotetext{
${ }^{64}$ Elie Zahar (1973) refers to the connection between special and general relativity as the criterion to decide the case of Einstein vs. Lorentz. However, this author does not argue in terms of non-consequential empirical evidence. Presupposing the correctness of Lakatos account of scientific progress in terms of increasing empirical and heuristic power of scientific research programs, he states that it was Einstein's research program what defeated Lorentz's theory. The argument presupposes that Einstein's road from special to general relativity can be described in terms of a Lakatosian research program, of course. I think that this presupposition is highly dubious. First, Zahar states that one of the heuristic principles that define Einstein's research program was the generalization of the principle of relativity by means of the attainment of general covariance. However, recent historiography on the formulation of general relativity shows that the quest for general covariance was a constraint that Einstein originally considered, then abandoned because of certain difficulties he found and which he expressed in the famous hole argument, and that he finally resumed - but only to realize that the physical meaning he had originally assigned to general covariance was unjustified. These zigzags in the relevance of the principle of general covariance make it dubious that its role in the formulation of general relativity can be described by means of Lakatos' concept of a scientific research program. Second, Zahar also states that the successful explanation of the behavior of Mercury's perihelion counts as a 'novel prediction' that testifies the empirical and heuristic power of Einstein's research program. Zahar's concept of 'novel prediction' asserts that even if the predicted fact was already known at the time of the formulation of the theory, the successful prediction was not a specific goal in the formulation of the theory. Again, recent historiography shows that the correct prediction regarding Mercury's perihelion was an explicit and specific goal that Einstein considered in the formulation of the theory. For example, when narrating the failure of the Entwurf theory, Walter Isaacson reports that 'Einstein hoped that his theory of relativity [the Entwurf], when its gravitational field equations were applied to the sun, would explain Mercury's orbit. Unfortunately, after a lot of calculations and corrected mistakes, he and Besso came up with a value of 18 seconds of an arc per century for how Mercury's perihelion should stray, which was not even halfway correct. The poor result convinced Einstein not to publish the Mercury calculations' (Isaacson 2007, p. 199). More generally, even though there is a continuity in Einstein's road from special to general relativity, it is quite difficult to make sense of it in terms of Lakatos' model. The nonconsequential evidence standpoint, on the other hand, does not require to accept any specific model for the rationality and development of science. Non-consequential evidence settles the matter independently of whether one is a Lakatosian, a Kuhnian, or what have you.
} 
of Einstein vs. Lorentz had been already decided. Therefore, the second standard of non-consequential evidence was historically irrelevant, it only works from a conceptual point of view.

Summarizing, we have that the accusations of ad-hocness on Lorentz's theory and recourse to the mathematical elegance of special relativity do not work as justified grounds to make a choice favoring Einstein's theory. The physical simplicity of special relativity that reflects in firmer ontological foundations, and the superfluous status of the ether in Lorentz's theory are features that do provide criteria according to which the former theory is superior. However, in general, non-empirical features are not sources for a full solution of the problem of empirical equivalence. Even though they provide rational grounds to prefer one theory in a predictively equivalent pair, this is not enough to justify a completely objective and uniquely determined choice. In the case of special relativity and Lorentz's theory, though, the inter-theoretical connections of the latter and quantum theory and of the former and general relativity are such that nonconsequential empirical evidence can decide the contend in a unique and objective way.

\section{REFERENCES}

Balashov, Y. \& Janssen, M. (2003). Presentism and relativity. British Journal for the Philosophy of Science, 54, 327346.

Boyd, R. (1973). Realism, underdetermination and a causal theory of evidence. Noûs, 7, 1-12.

Brown, H. (2001). The origins of length contraction: I. The Fitzgerald-Lorentz deformation hypothesis. American Journal of Physics, 69, 1044-1054.

Brown, H. (2003). Michelson, Fitzgerald and Lorentz: the origins of special relativity revisited. Bulletin de la Société des Sciences et des Lettres de Lódz, Volume LIII; Série: Reserches sur les Déformations, Volume XXXIX, 23-35.

Brown, H. (2005). Physical relativity: space-time structure from a dynamical perspective. Oxford: Oxford University Press.

Brown, H. \& Pooley. O. (2006). MinkowskisSpace-time: a glorious non-entity. In D. Dieks (Ed.), The ontology of spacetime (pp. 67-89), Amsterdam: Elsevier.

Brush, S. (1999). Why was relativity accepted? Physics in Perspective, 1, 184-214.

Bunge, M. (1961). The weight of simplicity in the construction and assaying of scientific theories. Philosophy of Science, 28, 120-141.

Darrigol, O. (1995). Henri Poincaré's criticism of fin de siècle electrodynamics. Studies in History and Philosophy of Modern Physics, 26, 1-44.

Dieks, D. (2009). Bottom-up versus top-down: the plurality of explanation and understanding in physics. In H. de Regt et al. (Eds.), Scientific understanding: philosophical perspectives (pp. 230-248). Pittsburgh: University of Pittsburgh Press.

Dorling, J. (1968). Length contraction and clock synchronization: the empirical equivalence of Einsteinian and Lorentzian theories. British Journal for the Philosophy of Science, 19, 67-69. 
Earman, J. (1989). World enough and spacetime: absolute versus relationist theories of space and time. Cambridge: MIT Press.

Einstein, A. (1919). My theory. The London Times, November 28. Reprinted in A. Einstein, Ideas and opinions. New York: Crown Publishers, 1954.

Einstein, A. (1959). Autobiographical notes. In P. Schilpp (Ed.), Albert Einstein: philosopher-scientist. New York: Harper.

Frisch, M. (2005). Mechanisms, principles, and Lorentz's cautious realism. Studies in History and Philosophy of Modern Physics, 36, 659-679.

Frisch, M. (2011). Principle or constructive relativity. Studies in History and Philosophy of Modern Physics, 42, 172183.

Galison, P. (1979). Minkowski’s space-time: from visual thinking to the absolute world. Historical Studies in the Physical Sciences, 10, 85-121.

Grünbaum, A. (1959). The falsifiability of the Lorentz-Fitzgerald contraction hypoythesis. British Journal for the Philosophy of Science, 10, 48-50.

Grünbaum, A. (1973). Philosophical problems of space and time. Dordrecht: Reidel.

Grünbaum, A. (1976). Ad hoc auxiliary hypotheses and falsificationism. British Journal for the Philosophy of Science, 27, 329-362.

Hempel, C. (1945). Studies in the logic of confirmation (II), Mind, 54, 97-121.

Hunt, B. (1988). The origins of the Fitzgerald contraction. British Journal for the Philosophy of Science, 21, 61-76.

Isaacson, W. (2007). Einstein: his life and universe. New York: Simon \& Schuster.

Ives, H. (1952). Derivation of the mass-energy relation. Journal of the Optical Society of America, 42, 540-543.

Janssen, M. (1995). A comparison between Lorentz's ether theory and special relativity in the light of the experiments of Trouton and Noble. PhD Dissertation.

Janssen, M. (2002a). Reconsidering a scientific revolution: the case of Einstein versus Lorentz. Physics in Perspective, $4,421-446$.

Janssen, M. (2002b). COI stories: explanation and evidence in the history of science. Perspectives on Science, 10, $457-$ 522 .

Janssen, M. (2003). The Trouton experiment, $E=m c^{2}$, and a slice of Minkowski space-time. In A. Ashtekar et al. (Eds), Revisiting the foundations of relativistic physics: Festschrift in honor of John Stachel (pp. 27-54). Dordrecht: Kluwer.

Janssen, M. (2009). Drawing the line between kinematics and dynamics in special relativity. Studies in History and Philosophy of Modern Physics, 40, 26-52.

Janssen, M. \& Mecklenburg, M. (2007). From classical to relativistic mechanics: electromagnetic models of the electron. In V. F. Hendricks et al. (Eds.), Interactions: mathematics, physics and philosophy 1860-1930 (pp. 65-134). Dordrecht: Springer. 
Kragh, H. (1999). Quantum generations: a history of physics in the twentieth century. Princeton: Princeton University Press.

Kox, A. J. (2013). Hendrik Antoon Lorentz's struggle with quantum theory. Archive for History of Exact Sciences, 67, 149-170.

Laudan, L. (1977). Progress and its problems: towards a theory of scientific growth. Berkeley: University of California Press.

Laudan, L. \& Leplin, J. (1991). Empirical equivalence and underdetermination. The Journal of Philosophy, 88, 449472 .

Lorentz, H. (1916). The theory of electrons and its applications to the phenomena of light and radiant heat. Leipzig: Teubner. Reprinted in facsimile by Dover: New York, 1952.

McAllister, J. (1989). Truth and beauty in scientific reason. Synthese, 78, 25-51.

McCormmach, R. (1970). H. A. Lorentz and the electromagnetic view of nature. Isis, 61, 459-497.

Miller, A. (1973). A study of Henri Poincaré's sur la dynamique de l'électron. Archive for History of Exact Sciences, 10, 207-328.

Miller, A. (1998). Albert Einstein's special theory of relativity: emergence (1905) and early interpretation (1905-1911), New York: Springer.

Norton, J. (2008). Must evidence underdetermine theory? In M. Howard \& J. Kourany (Eds.), The challenge of the social and the pressure of practice: science and values revisited. Pittsburgh: University of Pittsburgh Press.

Nugayev, R. (1985). The history of quantum mechanics as a decisive argument favoring Einstein over Lorentz. Philosophy of Science, 52, 44-63.

Okasha, S. (1997). Laudan and Leplin on empirical equivalence. British Journal for the Philosophy of Science, 48, 251256.

Poincaré, H. (1904). L'état actuel et l'avenir de la physique mathématique. Bulletin des Sciences Mathematiques, 28, translated in chapters 7-9 in H. Poincaré: The Value of Science, New York: Dover, 1958.

Popper, K. (1959). Testability and ad hocness of the contraction hypothesis. British Journal for the Philosophy of Science, 10,50 .

Popper, K. (2002). The logic of scientific discovery. London: Routledge.

Reichenbach, H. (1958). The philosophy of space \& time. New York: Dover.

Rindler, W. (1991). Introduction to special relativity. Oxford: Oxford University Press.

Sartori, L. (1996). Understanding relativity. Berkeley: University of California Press.

Schaffner, K. (1974). Einstein versus Lorentz: research programmes and the logic of comparative theory evaluation. British Journal for the Philosophy of Science, 25, 45-78.

Torretti, R. (1996). Relativity and geometry. New York: Dover.

Van Fraassen, B. (1980). The scientific image. Oxford: Clarendon Press. 
Walter, S. (2010). Minkowski’s modern world. In V. Petkov (Ed.), Minkowski spacetime: a hundred years later. New York: Springer.

Zahar, E. (1973). Why did Einstein's programme supersede Lorentz's? British Journal for the Philosophy of Science, 24, 95-123, 223-262. 